\title{
A Characterization of Wavelet Convergence in Sobolev Spaces
}

\author{
Mark A. Kon ${ }^{1}$ \\ Boston University \\ Louise Arakelian Raphael ${ }^{2}$ \\ Howard University
}

Dedicated to Prof. Robert Carroll on the occasion of his 70th birthday.

\begin{abstract}
We characterize uniform convergence rates in Sobolev and local Sobolev spaces for multiresolution analyses.
\end{abstract}

\section{Introduction and definitions}

In [KR1] it is shown that convergence rates of wavelet and multiresolution expansions depend on smoothness of the expanded function $f$. Specifically, if $s$ is not larger than a fixed parameter and $f \in H^{s}$ then the error of approximation is $O\left(2^{-n(s-d / 2)}\right)$, with $d$ dimension and $n$ the number of scales used in the expansion. This result is expected (see [Wa]) and comparable to Fourier approximation orders. In this paper we study a very different phenomenon which occurs for function spaces beyond a certain degree of smoothness. In these cases the rate of convergence "freezes" and fails to improve, no matter what the smoothness of $f$. Such behaviors have been studied in the context of approximation theory.

We show here that the smoothness level $s$ at which such freezing occurs depends on the wavelet or scaling function in a well-defined way, and that it more generally depends on the reproducing kernel of the multiresolution analysis (MRA). This completes a characterization of pointwise convergence rates in Sobolev spaces for general MRA's begun in [KR1], to include Sobolev spaces $H^{s}$ with $s$ large. In addition (Theorem 8), we extend these results to local Sobolev spaces which are related to spaces with uniformly bounded derivatives. See [Ma, Me, Wa] for some results on $L^{2}$ and $L^{\infty}$ convergence rates of $r$-regular multiresolution expansions.

\footnotetext{
${ }^{1}$ Research partially supported by the National Science Foundation, Air Force Office of Scientific Research, and U.S. Fulbright Commission

${ }^{2}$ Research partially supported by the Air Force Office of Scientific Research and the National Security Agnency
}

1991 AMS Mathematics Subject Classifications: primary 42C15; secondary 40A30 
In [KR1] it is assumed wavelets $\psi^{\lambda}$ or scaling functions $\phi$ have sufficient regularity that the regularity of $f$ is the limiting factor in convergence rates. Here we assume $f$ has sufficient regularity, and show that limitations on approximation rates then depend on regularity properties of $\psi^{\lambda}$ or $\phi$. We indicate more generally how the interaction of the regularities of $\psi^{\lambda}$ and of $f$ limits convergence. This complements results (see [D2, Ma, $\mathrm{Me}]$ ) which relate convergence rates for functions $f$ to exact characterizations of function spaces. These results rely on sufficient regularity for $\psi^{\lambda}$, and so do not give information when wavelets have lower regularity relative to $f$.

Our conditions on behavior of wavelets near the origin are more precise versions of Strang-Fix type conditions $[\mathrm{SF}]$. They can be translated into moment conditions on wavelets in the case that the moment powers are integers; see also [KR2].

For detailed definitions and theory of an MRA we refer to [D2]. An MRA is defined as an increasing sequence of subspaces $\left\{V_{n}\right\}$ of $L^{2}\left(\mathbb{R}^{d}\right)(d \geq 1)$ such that $f(x) \in V_{n}$ iff $f(2 x) \in V_{n+1}$, the intersection of the spaces is $\{0\}$, the closure of their union is all of $L^{2}$, and $V_{0}$ is invariant under translations of integers. It is also generally assumed (though we do not require it here) that there exists a function $\phi(x)$ (the scaling function) whose integer translates form an orthogonal basis for $V_{0}$.

Let $W_{i}$ be the orthogonal complement of $V_{i}$ in $V_{i+1}$, i.e., $W_{i}=V_{i+1} \ominus V_{i}$, so that $V_{i+1}=$ $V_{i} \oplus W_{i}$. From existence of $\phi$ it follows (see, e.g., [D2], [Me], [Wo]) that there is a set of basic wavelets $\left\{\psi^{\lambda}(x)\right\}_{\lambda \in \Lambda}$ (with $\Lambda$ a finite index set) such that $\psi_{j k}^{\lambda}(x) \equiv$ $2^{j d / 2} \psi^{\lambda}\left(2^{j} x-k\right) \quad\left(j \in \mathbb{Z}, k \in \mathbb{Z}^{\mathrm{d}}\right)$ form an orthonormal basis for $W_{j}$ for fixed $j$, and form an orthonormal basis for $L^{2}\left(\mathbb{R}^{\mathrm{d}}\right)$ as $\lambda, j, k$ vary.

Our results will hold for any wavelet set $\left\{\psi^{\lambda}\right\}_{\lambda}$ related to $W_{0}$ whose translations and dilations form an orthonormal basis for $L^{2}\left(\mathbb{R}^{\mathrm{d}}\right)$, regardless of how they are constructed (see [D2], Ch. 10; [Me]; [HW]).

It follows from the above definitions that there exist numbers $\left\{h_{k}\right\}_{k \in \mathbb{Z}^{d}}$ such that the scaling equation

$$
\phi(x)=2^{d} \sum_{k=-\infty}^{\infty} h_{k} \phi(2 x-k)
$$

holds. We define

$$
m_{0}(\xi) \equiv \sum_{k=-\infty}^{\infty} h_{k} e^{-i k \xi}
$$

to be the symbol of the MRA. Note it satisfies $\widehat{\phi}(\xi)=m_{0}(\xi / 2) \widehat{\phi}(\xi / 2)$, where ${ }^{-}$denotes Fourier transform. Our convention for the Fourier transform is

$$
\widehat{\phi}(\xi) \equiv \mathcal{F}(\phi)(\xi) \equiv(2 \pi)^{-d / 2} \int_{\mathbb{R}^{d}} \phi(x) e^{-i \xi \cdot x} d x
$$

where $\xi \cdot x=\xi x$.

Definitions 1.1: We define $P_{n}$ and $Q_{n}$ to be the $L^{2}$ orthogonal projections onto $V_{n}$ and $W_{n}$, respectively, with kernels (when they exist) $P_{n}(x, y)$ and $Q_{n}(x, y)$. We define $P_{0}=P$. 
Given $f \in L^{2}$,

(i) the multiresolution approximation of $f$ is the sequence $\left\{P_{n} f\right\}_{n}$;

(ii) the wavelet expansion of $f$ is

$$
\sum_{j ; k ; \lambda} a_{j k}^{\lambda} \psi_{j k}^{\lambda}(x) \sim f,
$$

with $a_{j k}^{\lambda}$ the $L^{2}$ expansion coefficients of $f$, and $\sim$ denoting convergence in $L^{2}$;

(iii) the scaling expansion of $f$ is

$$
\sum_{k} b_{k} \phi_{k}(x)+\sum_{j \geq 0 ; k ; \lambda} a_{j k}^{\lambda} \psi_{j k}^{\lambda}(x) \sim f,
$$

where the $b_{k}, a_{j k}^{\lambda}$ are $L^{2}$ expansion coefficients, and $\phi_{k}(x)=\phi(x-k)$.

We say such sums converge in any given sense (e.g., pointwise, in $L^{p}$, etc.) if the sums are calculated in such a way that at any stage in the summation there is a uniform bound on the range (largest minus smallest) of $j$ values for which we have only a partial sum over $k$, $\lambda$.

Definitions 1.2: A multiresolution analysis (MRA) or family of wavelets $\psi^{\lambda}$ yields pointwise order of approximation (or pointwise order of convergence) $s>0$ in $H^{r}$ if for any $f \in H^{r}$, the $j^{\text {th }}$ order approximation $P_{j} f$ satisfies

$$
\left\|P_{j} f-f\right\|_{\infty}=O\left(2^{-j s}\right),
$$

as $j$ tends to infinity, if $r-d / 2>0$ (if $r-d / 2 \leq 0$ the left side of (1.4) is in fact infinite for some $f$ ). It yields best pointwise order of approximation (or convergence) $s>0$ in $H^{r}$ if $s$ is the largest positive number such that (1.4) holds for all $f \in H^{r}$. If the supremum $s$ of the numbers for which (1.4) holds is not attained, then we denote the best pointwise order of convergence by $s^{-}$.

The MRA yields optimal pointwise order of approximation (or convergence) $s$ if $s$ is the best pointwise order of approximation for sufficiently smooth $f$, i.e. for $f \in H^{r}$ for sufficiently large $r$. Thus this order of convergence is the best possible order in any Sobolev space. We say $s=\infty$ if the best order of approximation in $H^{r}$ becomes arbitrarily large for large $r$.

By convention best order of approximation 0 means that the supremum in (1.4) fails to go to 0 ; thus $s \geq 0$ by our definitions.

We remark that our use of the term best approximation order differs from the term best approximation as used, e.g., by Singer [Si]. In addition the word best is used for technical reasons associated with the formulations of our statements. Specifically, in this paper an expansion has order of approximation $s$ if the optimal exponent in (1.4) is $s$ or better, while it has best order of approximation $s$ if the optimal exponent is $s$ and no larger than $s$.

Definitions 1.3: The Sobolev space $H^{\mathrm{s}}$ is defined by 


$$
H^{\mathrm{s}} \equiv\left\{f \in L^{2}\left(\mathbb{R}^{d}\right):\|f\|_{\mathrm{s}} \equiv \sqrt{\int|\hat{f}(\xi)|^{2}\left(1+|\xi|^{2}\right)^{\mathrm{s}} d \xi}<\infty\right\}
$$

The homogeneous Sobolev space is:

$$
H_{h}^{\mathrm{s}} \equiv\left\{f \in L^{2}\left(\mathbb{R}^{d}\right):\|f\|_{h, s} \equiv \sqrt{\int|\hat{f}(\xi)|^{2}|\xi|^{2 s} d \xi}<\infty\right\} .
$$

Note the spaces contain the same functions (by virtue of the fact that $H_{h}^{s}$ is restricted to $L^{2}$ ). Only the norms differ, and the second space is incomplete as defined (its completion contains non- $L^{2}$ functions which grow at $\infty$ ).

Definitions 1.4: A function $f(x)$ on $\mathbb{R}^{\mathrm{d}}$ is radial if $f$ depends on $|x|$ only. A real valued radial function is radial decreasing if $|f(x)| \leq|f(y)|$ whenever $|x| \geq|y|$. A function $f(x)$ is in the radially bounded class [RB] (c.f. [GK1,2]) if it is absolutely bounded by a positive $L^{1}$ radial decreasing function $\eta(x)$, i.e., $\eta\left(x_{1}\right)=\eta\left(x_{2}\right)$ when $\left|x_{1}\right|=\left|x_{2}\right|$, with $\eta\left(x_{1}\right) \leq \eta\left(x_{2}\right)$ whenever $\left|x_{1}\right| \geq\left|x_{2}\right|$, and $\eta(x) \in L^{1}\left(\mathbb{R}^{\mathrm{d}}\right)$ (note we assume $\eta$ is defined and finite at the origin, so that $f$ must be bounded).

Less general forms of the following two theorems were announced in [KKR2, KR2]; Theorems 1 through 4 were proved in [KR1] (see http://math.bu.edu/people/mkon/). These theorems say that under mild assumptions on the MRA (i.e., the scaling function or wavelets have a radially decreasing $L^{1}$ majorant), for $f \in H^{s}\left(\mathbb{R}^{d}\right)$, the rate of convergence to 0 of the error $\left\|f-P_{j} f\right\|_{\infty}$ has sharp order $2^{-j(s-d / 2)}$. We emphasize that the conditions in Theorems 1 through 4 are equivalent.

Theorem 1 [KR1]: Given a multiresolution analysis with either

(i) a scaling function $\phi \in[\mathrm{RB}]$,

(ii) basic wavelets $\psi^{\lambda} \in[\mathrm{RB}]$ or

(iii) a kernel $P(x, y)$ for the basic projection $P$ satisfying $|P(x, y)| \leq H(x-y)$ with $H \in[\mathrm{RB}]$, then the following conditions ( $a$ to $b$ ) are equivalent for $s>d / 2$, with $d$ the dimension:

(a) The multiresolution approximation yields pointwise order of approximation $s-d / 2$ in $H^{\mathrm{s}}$.

$\left(a^{\prime}\right)$ The multiresolution approximation yields best pointwise order of approximation $s-d / 2$ in $H^{\mathrm{s}}$.

$\left(a^{\prime \prime}\right)$ The multiresolution approximation yields best pointwise order of approximation $r-d / 2$ in $H^{r}$ for all $d / 2<r \leq s$.

(b) The projection $I-P_{n}: H_{h}^{s} \rightarrow L^{\infty}$ is bounded, where $I$ is the identity.

Theorem 2 is related to the vanishing moments property of the wavelets $\psi^{\lambda}$.

Theorem 2 [KR1]: Under the assumptions of Theorem 1, if there exists a family $\left\{\psi^{\lambda}\right\}$ of basic wavelets corresponding to $\left\{P_{n}\right\}$ with $\psi^{\lambda}(x) \in[\mathrm{RB}]$, then the following conditions are equivalent to those of Theorem 1: 
(c) For every such family of basic wavelets and each $\lambda$, $\psi^{\lambda} \in H_{h}^{-s}$, the dual of $H_{h}^{s}$.

$\left(c^{\prime}\right)$ For every such family of basic wavelets and for each $\lambda$,

$$
\int_{|\xi|<\delta}\left|\hat{\psi^{\lambda}}(\xi)\right|^{2}|\xi|^{-2 s} \mathrm{~d} \xi<\infty
$$

for some (or for all) $\delta>0$.

(c") For some such family of basic wavelets, (1.5a) holds.

Definition 1.5: We define the space $\mathcal{F} H^{s}$ to be the Fourier transforms of functions in $H^{s}$, with the analogous definition for $\mathcal{F} H_{h}^{s}$.

Theorems 3 and 4 are related to the so-called Strang-Fix conditions on the scaling function and the low pass filter $m_{0}$.

Theorem 3 [KR1]: Under the assumptions of Theorem 1, if there exists a scaling function $\phi$ corresponding to $\left\{P_{n}\right\}$, the following conditions are equivalent to those of Theorems 1 and 2:

(d) For every such scaling function, $1-(2 \pi)^{d / 2}|\widehat{\phi}| \in \mathcal{F} H_{h}^{-s}$.

$\left(d^{\prime}\right)$ For every scaling function $\phi \in[\mathrm{RB}]$ corresponding to $\left\{P_{n}\right\}$

$$
\int_{|\xi|<\delta}\left(1-(2 \pi)^{d / 2}|\hat{\phi}(\xi)|\right)|\xi|^{-2 s} \mathrm{~d} \xi<\infty
$$

for some (or all) $\delta>0$.

$\left(d^{\prime \prime}\right)$ For some scaling function $\phi$ corresponding to $\left\{P_{n}\right\},(1.5 b)$ holds.

$\left(d^{\prime \prime \prime}\right)$ For every scaling function $\phi \in[\mathrm{RB}]$ corresponding to $\left\{P_{n}\right\}$

$$
\int_{|\xi|<\delta} \sum_{\ell \neq 0}|\hat{\phi}(\xi+2 \pi \ell)|^{2}|\xi|^{-2 s} d \xi<\infty
$$

We define $F=\{0,1\}^{\mathrm{d}}$ to be the set of all $d$-vectors with entries from the pair $\{0,1\}$.

Theorem 4 [KR1]: If $m_{0}(\xi)$ is a symbol of a multiresolution expansion corresponding to a sequence of projections $P_{n}$ as in Theorem 1, the following conditions are equivalent to those in Theorems 1-3 for $s>d / 2$ :

(e) For every symbol $m_{0}(\xi)$ corresponding to $\left\{P_{n}\right\}$,

$$
\int_{|\xi|<\delta}\left(1-\left|m_{0}(\xi)\right|^{2}\right)|\xi|^{-2 s} d \xi<\infty
$$

for some (or all) $\delta>0$

$\left(e^{\prime}\right)$ For some symbol $m_{0}(\xi)$ corresponding to $\left\{P_{n}\right\},(1.5 d)$ holds.

$\left(e^{\prime \prime}\right)$ Every (or some) symbol $m_{0}(\xi)$ corresponding to $\left\{P_{n}\right\}$ satisfies 


$$
\int_{|\xi-\pi \epsilon|<\delta}\left|m_{0}(\xi)\right|^{2}|\xi-\pi \epsilon|^{-2 s} d \xi<\infty
$$

for some (or all) $\delta>0$ and for every $\epsilon \in F^{\prime}$, where we define

$$
F^{\prime} \equiv F \backslash\{0\},
$$

where $F=\{0,1\}^{d}$ and 0 denotes the zero vector in (1.6).

For the remainder of the paper, we assume the following:

Assumptions: We assume in all of the following theorems that one of the following holds:

(i) The projection $P$ onto $V_{0}$ satisfies

$$
|P(x, y)| \leq H(x-y) \text { for some } H \in[\mathrm{RB}] .
$$

If a scaling function $\phi$ exists, (ii) $\phi \in[\mathrm{RB}]$.

If a wavelet family $\psi^{\lambda}$ exists, (iii) $\psi^{\lambda}(x)(\ln (2+|x|) \in[\mathrm{RB}]$ for all $\lambda$.

Remark: It is shown in [KKR1] that $(i i) \Rightarrow(i)$ and $(i i i) \Rightarrow(i)$. This follows from the representations of $P(x, y)$ in terms of sums involving $\phi$ or $\psi^{\lambda}$ when they exist.

Note that the condition on $\psi$ in $(i i i)$ is somewhat stricter than that required for Theorems 1 through 4 above. It is required for existence of a kernel $P_{j}(x, y)$ for the projection $P_{j}$ satisfying $\left|P_{j}(x, y)\right| \leq H(x-y)$, with $H(\cdot)$ a radial decreasing $L^{1}$ function. This class of wavelets includes all $r$-regular wavelets (see $[\mathrm{Me}]$ ) for any $\mathrm{r} \geq 0$. The assumptions are also needed for appropriate $L^{p}$ and a.e. convergence properties of wavelet expansions [KKR1].

Theorems 1 - 4 apply only to expansions of functions in Sobolev spaces $H^{s}$ for which $E=I-P_{0}: H_{h}^{s} \rightarrow L^{\infty}$ is bounded (see (b) of Theorem 1). They say nothing about the case of unbounded $E$. We show here that for larger $s$ (for which $E$ is unbounded), approximation rates are essentially the same as for the largest $s$ for which $E$ is bounded. Details of the approximation rates, however, depend somewhat delicately on the wavelets or scaling function. Before giving an overview of our new results in Theorem 5, we refer the reader to formulas $(1.5 \mathrm{a}, \mathrm{b}, \mathrm{d})$ as motivation for the following definition.

Definitions 1.6: We define for $s, c \geq 0$ 


$$
\begin{aligned}
& I_{s}(c) \equiv \int_{1 \geq \xi \mid \geq c}\left(1-(2 \pi)^{d / 2}|\hat{\phi}(\xi)|\right)|\xi|^{-2 s} \mathrm{~d} \xi \\
& K_{s}(c) \equiv \sup _{\lambda} \int_{1 \geq|\xi| \geq c}\left|\widehat{\psi}^{\lambda}(\xi)\right|^{2}|\xi|^{-2 s} \mathrm{~d} \xi \\
& L_{s}(c) \equiv \int_{1 \geq|\xi| \geq c}\left(1-\left|m_{0}(\xi)\right|^{2}\right)|\xi|^{-2 s} \mathrm{~d} \xi .
\end{aligned}
$$

In this paper an often-used consequence of Theorems 1 through 4 is the existence of a least upper bound $\sigma$ (best Sobolev parameter), depending only on the MRA, for which $(b)$ of Theorem 1 holds. This motivates the following definition.

Definition 1.7: The best Sobolev parameter $\sigma$ of an MRA is

$$
\sigma=\sup \left\{s>0 \mid(I-P): H_{h}^{s} \rightarrow L^{\infty} \text { is bounded }\right\} .
$$

By convention $\sigma=0$ if the set in the supremum is empty. Some bounds on $\sigma$ follow from Theorem 1 above:

Proposition 1.8: If the best Sobolev parameter $\sigma \neq 0$, then $\sigma>d / 2$, and the set

$$
\Sigma \equiv\left\{s>0 \mid(I-P): H_{h}^{s} \rightarrow L^{\infty} \text { is bounded }\right\}
$$

satisfies

$$
\Sigma=(d / 2, \sigma] \quad \text { or } \quad \Sigma=(d / 2, \sigma) \text {. }
$$

Proof: Assume $\sigma \neq 0$, so that $\sigma>0$ (recall $\sigma=0$ means there is no positive order of convergence). Under any one of the assumptions (1.7), the kernel $P(x, y)$ of the projection onto $V_{0}$ is bounded by $K(x-y)$, with $K \in[\mathrm{RB}]$. Thus $K$ is bounded and in $L^{1}$, and hence $K(x-y) \in L^{2}[y]$ and is bounded in $L^{2}[y]$, uniformly in $x$. Thus for $f \in L^{2}$, Pf $\in L^{\infty}$.

But for every nonnegative integer $d$, there exist unbounded $f \in H_{h}^{d / 2}$. For such $f$, $E f=(I-P) f=f-P f$ is not in $L^{\infty}$, and so $E: H_{h}^{d / 2} \rightarrow L^{\infty}$ is unbounded. Similarly, $E: H_{h}^{s} \rightarrow L^{\infty}$ is unbounded for $0 \leq s \leq d / 2$. Thus if $\sigma \neq 0$, i.e., if $E$ is bounded for some $s>0$, then $E$ must be bounded for an $s>d / 2$. Therefore $\sigma$, the supremum of $s$ for which $E$ is bounded, must satisfy $\sigma>d / 2$.

To prove statement (1.8) we need only show $\Sigma$ is connected. This on the other hand follows by the equivalence of $\left(a^{\prime}\right),\left(a^{\prime \prime}\right)$, and $(b)$ of Theorem 1 , showing $\Sigma=(d / 2, \sigma)$ or $\Sigma=(d / 2, \sigma]$.

From Theorems 1 and 4 we then have immediately

Proposition 1.9: If the best Sobolev parameter $\sigma \neq 0$, then 


$$
\sigma=\sup \left\{s>0 \mid I_{s}(0)<\infty\right\}=\sup \left\{s>0 \mid K_{s}(0)<\infty\right\}=\sup \left\{s>0 \mid L_{s}(0)<\infty\right\} .
$$

In Theorems 1-4, $\sigma$ is important in that all statements hold only if $s \leq \sigma$. For approximation rates in $H^{s}$, we prove the following theorem. This summarizes convergence rates in all $H^{s}$ in terms of properties of the projections $P_{n}$ or of integrals involving the wavelets or scaling functions.

Theorem 5: Given a multiresolution approximation $\left\{P_{n}\right\}$,

(o) If $\sigma=0$, there is no positive order of approximation for the $\operatorname{MRA}\left\{P_{n}\right\}$ in any $H^{s}$, $s \in \mathbb{R}$.

If $(o)$ does not hold then $\sigma>d / 2$ and:

(i) For $0 \leq s \leq d / 2$, the best pointwise order of approximation in $H^{s}$ is 0 ;

(ii) If $d / 2<s<\sigma$, the best pointwise order of approximation in $H^{s}$ is $r=s-d / 2$;

(iii) If $s=\sigma$, the best pointwise order of approximation in $H^{s}$ is $r= \begin{cases}\sigma-d / 2 & \text { if } I_{\sigma}(0)<\infty \\ (\sigma-d / 2)^{-} & \text {if } I_{\sigma}(0)=\infty\end{cases}$

(iv) If $s>\sigma$, the best pointwise order of approximation in $H^{s}$ is $r=\left\{\begin{array}{ll}\sigma-d / 2 & \text { if } I_{\sigma+1 / 2}(c)=O(1 / c) \quad(c \rightarrow 0) \\ (\sigma-d / 2)^{-} & \text {otherwise }\end{array} ;\right.$

(v) In (iii) and (iv) above, $I_{s}(c)$ can be replaced by $K_{s}(c)$ or by $L_{s}(c)$.

Another way to say (iv) is that if $s>\sigma$, then there exists $g \in H^{s}\left(\mathbb{R}^{d}\right)$ such that $\sup 2^{j(s-d / 2)}\left\|g-P_{j} g\right\|_{\infty}=\infty$. This says the convergence rate cannot be improved for $j$

functions belonging even to very smooth Sobolev spaces. Moreover we note that the value $\sigma+1 / 2$ used above in (iv) is not crucial for its statement. Equivalent conditions to those in (iv) exist in the form $I_{\sigma+\alpha / 2}(c)=O\left(c^{-\alpha}\right)$ for any (or all) $\alpha>0$.

In terms of the Sobolev order $s$ of the expanded function $f$ and the best Sobolev parameter $\sigma$ of the MRA, the following diagram gives rates for an MRA expansion in any Sobolev space (or local Sobolev space; see below). The rates on the boundary region $s=\sigma$ in (iii) above are not indicated in the diagram. 


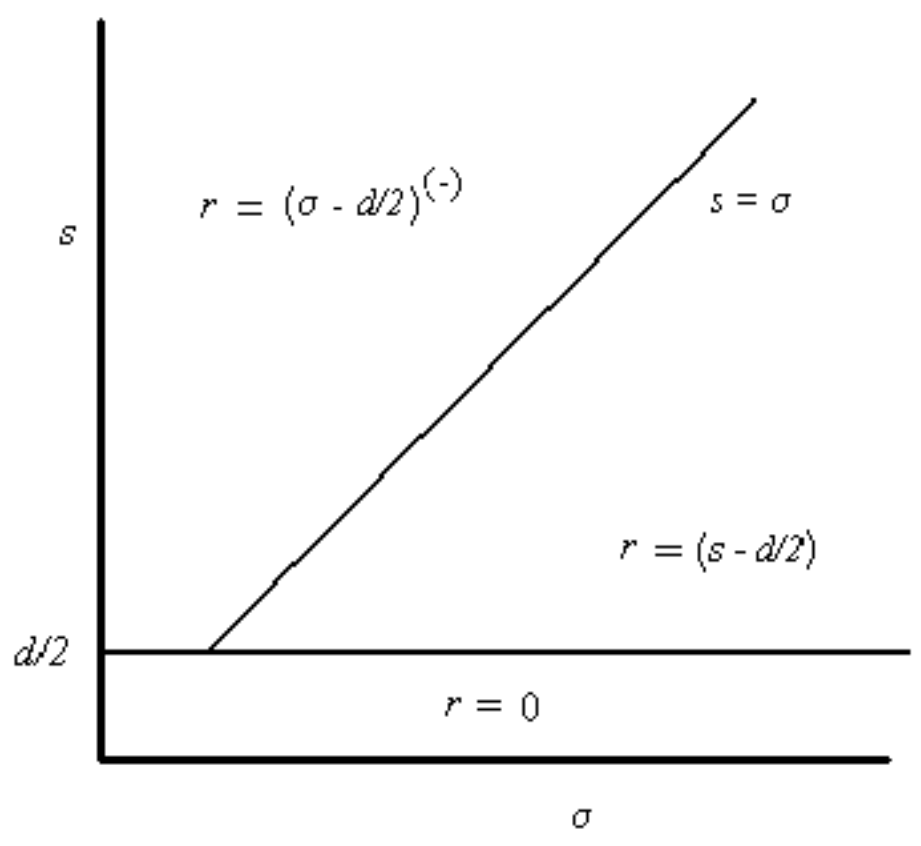

Figure: Approximation rate diagram; see Theorem 3(iii) for rates on the boundary $s=\sigma$. The $(-)$ in $(\sigma-d / 2)^{(-)}$indicates that the superscript - is present only in some cases.

We will show that this diagram applies to expansions of functions $f$ in $H^{s}$ and in uniform local spaces $H_{u l}^{s}$, when the decay rate $t$ of the scaling function satisfies $t-d \geq s$ (see Theorem 8). In addition, on compact subsets the rates in the diagram apply to functions in local Sobolev spaces $H_{l o c}^{s}$, when the wavelet has compact support. See Definitions 1.10 below for definitions of the spaces $H_{u l}^{s}$ and $H_{l o c}^{s}$.

We now establish several equivalent conditions for failure of convergence in all Sobolev spaces.

Corollary 6: The following ((a) through (e)) are equivalent for the $\operatorname{MRA}\left\{P_{n}\right\}$ :

(a) $I-P: H_{h}^{s} \rightarrow L^{\infty}$ is unbounded for all $s \geq 0$.

(b) This operator is unbounded for $s \in(d / 2, d / 2+\epsilon)$ for some $\epsilon>0$.

If there exists a family of wavelets $\left\{\psi^{\lambda}\right\}$ :

(c) For every $s>d / 2, \int\left|\widehat{\psi}^{\lambda}(\xi)\right|^{2}|\xi|^{-2 s} d \xi=\infty$ for some $\lambda$.

If there exists a scaling function $\phi$ :

(d) For every $s>d / 2, \int\left(1-(2 \pi)^{d / 2} \widehat{\phi}(\xi)\right)|\xi|^{-2 s} d \xi=\infty$.

(e) In every Sobolev space $H^{s}$ of nonnegative order, the MRA fails to have any positive order of convergence, i.e., the optimal order of convergence is 0.

We now state our results for optimal pointwise orders of convergence in Sobolev spaces. Recall $\sigma$ denotes the best Sobolev parameter of $\left\{P_{n}\right\}$, and that optimal order of approximation denotes the highest order of approximation in sufficiently smooth Sobolev spaces. 
Corollary 7: If the best Sobolev parameter $\sigma \neq 0$, then the wavelet collection $\psi^{\lambda}$ [or scaling function $\phi]$ yields optimal pointwise order of approximation $\sigma-d / 2$ if $I_{\sigma+1 / 2}(c)=O(1 / c)$ [where $I$ can be replaced by $K$ or $\left.L\right]$, and $(\sigma-d / 2)^{-}$otherwise. This optimal order is attained for all functions $f$ with smoothness greater than $\sigma$, i.e., for $f \in H^{s}$ with $s>\sigma$

Corollary 7 gives "best possible" pointwise convergence rates, i.e., convergence rates for the smoothest possible functions. In fact this optimal rate in fact is largely independent of how smoothness is defined, i.e., which particular scale of spaces we are working with. Such a statement is possible because when the smoothness parameter $s$ is sufficiently large, the most used scales of "smoothness spaces" satisfy inclusion relations. For example for $s^{\prime}$ large the space $H^{s^{\prime}}$ is contained in the sup-norm Sobolev space $L_{s}^{\infty}$ and in other $L^{\infty}$-type Sobolev spaces. Therefore the optimal rates of convergence given here are upper bounds for convergence rates in all $L_{s}^{\infty}$ spaces, no matter how smooth.

What is most important is that such inclusions work in both directions for the uniformly local spaces in Definitions 1.10 below. For example, for sufficiently large $r$, the uniformly local $L^{p}$-Sobolev space $L_{r, u l}^{p}$ is contained in spaces in the scale $\left\{L_{s, u l}^{q}\right\}_{s}$ for any fixed values of $p$ and $q$ (including $\infty$ ). In addition, for sufficiently large $s$ the space $L_{r, u l}^{p}$ contains Sobolev spaces $L_{s}^{q}$. This includes $L_{s}^{\infty}$ and its related smoothness spaces of functions with bounded derivatives.

This observation can then be used as follows. If $\phi(x)$ has decay rate $t$ (Def. 1.10) with $t-d>\sigma-d / 2$ (which holds for many wavelets of interest), then by Theorem 8 below the optimal convergence rate in all of the scales of uniformly local spaces $L_{s, u l}^{p}$ (including $p=\infty)$, is either $\sigma-d / 2$ or $(\sigma-d / 2)^{-}$, i.e., the same as in Corollary 7. Now the extension of Corollary 7 to the spaces $L_{r, u l}^{p}$ (Corollary 9) can be broadened, by the above argument, to more general scales of smoothness spaces, including smoothness spaces based on sup norms. (The caveat, however, is the scaling function $\phi$ must have sufficiently rapid decay.)

With this motivation, we now give the results for uniformly local Sobolev spaces. Our results will also extend to local Sobolev spaces $H_{l o c}^{s}$ with some caveats.

Definitions 1.10: The decay rate of a function $\phi$ is

$$
\sup \left\{t:|\phi(x)| \leq K|x|^{-t} \text { for some } K>0\right\} \text {. }
$$

We will assume here our decay rates $t$ are positive unless otherwise specified.

The local Sobolev space $H_{l o c}^{s}$ is $\left\{f: f \eta \in H^{s} \forall \eta \in C_{c}^{\infty}\right\}$, where $C_{c}^{\infty}$ is compactly supported $C^{\infty}$ functions. The uniform local Sobolev space $H_{u l}^{s}$ is $\left\{f:\|f\|_{s}^{u l}<\infty\right\}$, where the uniformly local norm $\|\cdot\|_{s}^{u l}$ is defined by (here $B_{x}$ is the unit ball centered at $x$ ):

$$
\|f\|_{s}^{u l} \equiv \sup _{x \in \mathbb{R}^{d}}\|f\|_{s, B_{x}}
$$

Above, the local norm is defined by 


$$
\|f\|_{s, B_{x}} \equiv \inf _{\left.f^{*}\right|_{B_{x}}=f, f^{*} \in H^{s}}\left\|f^{*}\right\|_{s} .
$$

Similarly, the space $L_{s}^{p} \equiv\left\{f \in L^{p}:(1-\Delta)^{s / 2} f \in L^{p}\right\}$ has a local version $L_{s, u l}^{p}$ defined analogously to the above with the norm $\left\|f^{*}\right\|_{s}$ in (1.9) replaced by the norm of the Sobolev space $L_{s}^{p}$.

Thus $H_{u l}^{s}$ consists of functions locally in $H^{s}$ with local $H^{s}$ norms uniformly bounded. The following results for $H_{u l}^{s}$ are effectively local versions of our rates of convergence results, modulo the spatial uniformity assumptions on functions in $H_{u l}^{s}$. Such uniformity assumptions also hold, e.g., for $L^{\infty}$ Sobolev spaces.

We require our working spaces $H_{u l}^{s}$ to have uniformly bounded local $L^{2}$ Sobolev norms rather than $L^{\infty}$ Sobolev norms, since the latter would make our work more difficult. As shown above, however, most other scales of smoothness spaces based on uniform $\left(L^{\infty}\right.$ type) bounds satisfy inclusion relations with the uniform Sobolev spaces $H_{u l}^{s}$, extending optimal convergence rate results to these spaces. Additionally, our results of course become entirely local (valid for local Sobolev spaces) if wavelets involved have compact support.

Recall from the definitions that approximation order 0 in a space $X$ means the error $E_{n} f$ fails to have any positive rate of decay for some $f \in X$.

Theorem 8 (Localization): The multiresolution or wavelet expansion corresponding to a scaling function $\phi \in[\mathrm{RB}]$ has a best pointwise approximation order of at least $\min (r, t-d)$ in $H_{u l}^{s}$, with $r$ the rate of best approximation in $H^{s}$ and $t>d$ the decay rate of $\phi$.

Corollary 9: If the best Sobolev parameter $\sigma \leq t-d / 2$ (where $t$ is the decay rate of $\phi$ ), then

(a) The optimal approximation order in the scale of spaces $H_{u l}^{s}$ is exactly $\sigma-d / 2$ if $I_{\sigma+1 / 2}(c)=O(1 / c)$ [where $I$ can be replaced by $K$ or $L$ ], and $(\sigma-d / 2)^{-}$otherwise.

(b) The same exact optimal approximation order holds in the scale of uniform local spaces $L_{s, u l}^{p}$ for fixed $1 \leq p \leq \infty$, and in particular also in the scale $L_{s, u l}^{\infty}$ and thus $L_{s}^{\infty}$.

Indeed, note that when $s$ is an even integer (i.e. the operator $(-\Delta)^{s / 2}$ is local) the spaces $L_{s}^{\infty}$ and $L_{s, u l}^{\infty}$ are identical, since the first space always is contained in the second, and if $f \notin L_{s}^{\infty}$, then there is a sequence of unit balls $B_{i}$ for which $\sup _{x \in B_{i}}(-\Delta)^{s / 2} f$ is unbounded, so that $f \notin L_{s, u l}^{\infty}$. Thus for each $s$, the scale $\left\{L_{r, u l}^{\infty}\right\}_{r}$ is eventually contained in $L_{s}^{\infty}$ for $r$ sufficiently large, and similarly $\left\{L_{r}^{\infty}\right\}_{r}$ is eventually in $L_{s, u l}^{\infty}$, so the two scales have identical optimal orders of convergence. This type of inclusion also works for other scales of $L^{\infty}$ Sobolev spaces, yielding identical optimal orders of approximation. 
Proposition 10: If $\phi$ is compactly supported, the best pointwise approximation rate for the expansion of any $f \in H_{\text {loc }}^{s}$ on any compact $K \subset \mathbb{R}^{d}$ is the same as the rate for the global space $H^{s}$.

Examples: To illustrate these results we give applications to some well-known wavelet approximations.

\section{Haar wavelets}

We calculate the exact approximation order for Haar wavelets. The scaling function $\phi$ is the characteristic function of the unit interval, whose Fourier transform is

$$
\widehat{\phi}(\xi)=\frac{1}{\sqrt{2 \pi}} \int_{0}^{1} e^{-i \xi x} d x=\frac{e^{-i \xi / 2}}{(\xi / 2) \sqrt{2 \pi}} \sin (\xi / 2)
$$

In this case $|\widehat{\phi}(\xi)|=\frac{1}{\sqrt{2 \pi}}+O\left(|\xi|^{2}\right)$ so by Proposition $1.9, \quad \sigma=3 / 2$, and $I_{\sigma}(0)=\infty$. In addition

$$
\begin{aligned}
I_{\sigma+1 / 2}(c) & =\int_{1>|\xi|>c}\left(1-(2 \pi)^{1 / 2}|\widehat{\phi}(\xi)|\right)|\xi|^{-2 \sigma-1} d \xi \\
& =\int_{1>|\xi|>c}\left(1-2 \frac{\sin \xi / 2}{\xi}\right)|\xi|^{-4} d \xi \\
& =\int_{1>|\xi|>c}\left(\xi^{2} / 24+O\left(\xi^{4}\right)\right) \xi^{-4} d \xi=O\left(c^{-1}\right) .
\end{aligned}
$$

Thus by Theorem 5, in $H^{s}$ Haar expansions have best order of convergence

$$
r=\left\{\begin{array}{ll}
0, & s \leq 1 / 2 \\
s-1 / 2, & 1 / 2<s<3 / 2 \\
1^{-}, & s=3 / 2 \\
1, & s>3 / 2
\end{array},\right.
$$

with the same orders in the uniform local Sobolev spaces $H_{u l}^{s}$ by Theorem 8 . By the same theorem, since $\phi$ is compactly supported, these orders of convergence to $f(x)$ hold uniformly for $x$ in a compact set, for any $f(x)$ locally in $H^{s}$. Finally by Corollary 7 , the optimal approximation order for such expansions (i.e., for arbitrarily smooth functions) is 1. By Corollary 9 this optimal order also holds, for example, in the scale $L_{s}^{\infty}$ of $L^{\infty}$ Sobolev spaces.

\section{Meyer wavelets}

We now consider standard Meyer wavelet expansions. The Fourier transform of the scaling function is [D2, page 137] 


$$
\widehat{\phi}(\xi)=\left\{\begin{array}{lc}
(2 \pi)^{-1 / 2}, & |\xi| \leq 2 \pi / 3 \\
(2 \pi)^{-1 / 2} \cos \left[\frac{\pi}{2} \nu\left(\frac{3}{4 \pi}|\xi|-1\right)\right], & 2 \pi / 3 \leq|\xi| \leq 4 \pi / 3 \\
0 & \text { otherwise }
\end{array}\right.
$$

where $\nu$ is an appropriately chosen smooth function for which $\widehat{\phi} \in C_{0}^{\infty}$. In this case $\sigma=\infty^{-}$, so we have order of convergence $s-1 / 2$ in each Sobolev space $H^{s}, s>1 / 2$, and convergence order 0 for $s \leq 1 / 2$. Note this implies that for functions in the intersection $\bigcap_{s} H^{s}$ of all Sobolev spaces, we have convergence faster than any finite order $r$. The same holds in the uniform local spaces $H_{u l}^{s}$ by Theorem 8 . Thus the optimal order of convergence in both these cases is $\infty$, i.e., convergence rates have no intrinsic limitations based on the wavelet for very smooth $f$.

\section{Battle Lemarié wavelets}

Consider now Battle-Lemarié wavelets, which effectively yield spline expansions of a given order. For splines of order 1 the B-spline is

$$
\phi(x)=\left\{\begin{array}{cc}
1-|x|, & 0 \leq|x| \leq 1 \\
0 & \text { otherwise }
\end{array} .\right.
$$

The Fourier transform is

$$
\widehat{\phi}(\xi)=(2 \pi)^{-1 / 2}\left(\frac{\sin \xi / 2}{\xi / 2}\right)^{2}
$$

Here $\phi$ is not a scaling function, since it does not have orthonormal translates. The orthogonalization trick ([D2], section 5.4) yields a scaling function $\tilde{\phi}$ with orthogonal translates, whose Fourier transform is

$$
\widehat{\widetilde{\phi}}(\xi)=\sqrt{3}(2 \pi)^{-1 / 2} \frac{4 \sin ^{2} \xi / 2}{\xi^{2}\left[1+2 \cos ^{2} \xi / 2\right]^{1 / 2}} .
$$

The corresponding wavelet has Fourier transform

$$
\widehat{\psi}(\xi)=\sqrt{3}(2 \pi)^{-1 / 2} e^{i \xi / 2} \sin ^{2} \xi / 4\left[\frac{1+2 \sin ^{2} \xi / 4}{1+2 \cos ^{2} \xi / 2}\right]^{1 / 2}\left[\frac{16 \sin ^{2} \xi / 4}{\xi^{2}\left[1+2 \cos ^{2} \xi / 4\right]^{1 / 2}}\right]=O\left(\xi^{2}\right) .
$$

From this it follows from Proposition 1.9 that $\sigma=5 / 2$. Further, $K_{\sigma}(0)=\infty$, while

$$
K_{\sigma+1 / 2}(c)=K_{3}(c)=\int_{|\xi|>c}|\widehat{\psi}(\xi)|^{2}|\xi|^{-6} d \xi=O(1 / c) \text {. }
$$

By Theorem 5, Battle-Lemarié expansions (and of course order one spline expansions, since the scaling spaces $V_{j}$ are the same) have order of convergence 


$$
r= \begin{cases}0, & s \leq 1 / 2 \\ s-1 / 2, & 1 / 2<s<5 / 2 \\ 2^{-}, & s=5 / 2 \\ 2, & s>5 / 2\end{cases}
$$

in $H^{s}$. In the uniform local spaces $H_{u l}^{s}$ the same approximation rates hold by Theorem 8 .

Analogous results hold for the higher order versions of these spline wavelets, and the corresponding spline expansions.

\section{Daubechies wavelets}

For standard Daubechies wavelets of order 2, we consider the symbol $m_{0}(\xi)$ (see (1.2); note the definition of the coefficients $h_{k}$ in equation (1.1)):

$$
\begin{aligned}
m_{0}(\xi) & =\frac{1}{8}\left[(1+\sqrt{3})+(3+\sqrt{3}) e^{-i \xi}+(3-\sqrt{3}) e^{-2 i \xi}+(1-\sqrt{3}) \mathrm{e}^{-3 i \xi}\right] \\
& =\frac{1}{8}\left[a+b z+c z^{2}+d z^{3}\right]
\end{aligned}
$$

Here $z=e^{-i \xi}$, and

$$
a=1+\sqrt{3}, b=3+\sqrt{3}, c=3-\sqrt{3}, d=1-\sqrt{3} .
$$

Note $m_{0}(0)=1$, while

$$
\begin{aligned}
& \left|m_{0}(\xi)\right|^{2} \\
& =\frac{1}{64}\left[\left(a^{2}+b^{2}+c^{2}+d^{2}\right)+2(a b+b c+c d) \cos \xi+2(a c+b d) \cos 2 \xi+2 a d \cos 3 \xi\right] .
\end{aligned}
$$

Therefore $\left.\frac{d}{d \xi}\left|m_{0}(\xi)\right|^{2}\right|_{\xi=0}=0$. Since $a c+b d=0, \quad a b+b c+c d=18, \quad$ and $a d=-2$,

$$
\frac{d^{2}}{d \xi^{2}}\left|m_{0}(\xi)\right|^{2}=-\frac{1}{64}[2(a b+b c+c d) \cos \xi+18 a d \cos 3 \xi]=-\frac{1}{64}[36 \cos \xi-36 \cos 3 \xi]
$$

and so $\left.\frac{d^{2}}{d \xi^{2}}\left|m_{0}(\xi)\right|^{2}\right|_{\xi=0}=0$. In addition, $\left.\frac{d^{3}}{d \xi^{3}}\left|m_{0}(\xi)\right|^{2}\right|_{\xi=0}=0$ but

$$
\frac{d^{4}}{d \xi^{4}}\left|m_{0}(\xi)\right|^{2}=\frac{1}{64}[36 \cos \xi-324 \cos 3 \xi]
$$

so $\left.\frac{d^{4}}{d \xi^{4}}\left|m_{0}(\xi)\right|^{2}\right|_{\xi=0} \neq 0$. Therefore $\left|m_{0}(\xi)\right|^{2}=1+O\left(|\xi|^{4}\right) \quad(\xi \rightarrow 0)$, so Theorem 4 implies $\sigma=5 / 2$. Thus by Theorem 5 , in $H^{s}$ the best order of approximation for these Daubechies wavelets is 


$$
r=\left\{\begin{array}{ll}
0, & s \leq 1 / 2 \\
s-1 / 2, & 1 / 2<s<5 / 2 \\
2^{-}, & s=5 / 2 \\
2, & s>5 / 2
\end{array} .\right.
$$

Similar analyses can of course be done for higher order Daubechies expansions. As before, by Theorem 8 , the global space $H^{s}$ can be replaced by the uniform local space $H_{u l}^{s}$. We see the optimal order of convergence for Daubechies wavelets of order 2 is 2 .

For the compactly supported Daubechies order 2 wavelets, these are entirely local results. Thus for any $f \in H_{l o c}^{s}$, the above exact approximation rates hold uniformly on any compact $K \subset \mathbb{R}^{d}$.

Remark: Our results imply that for one dimensional [RB] scaling functions with $C^{\infty}$ Fourier transforms (e.g., for compactly supported ones), optimal orders of convergence are always integers. The reason is clear from Theorem 5, since $\sigma$ for such $\phi$ is always a halfinteger (see Def. 1.6 and Prop. 1.9), given the Fourier transform $\widehat{\phi}$ is always infinitely differentiable at the origin. Specifically $|\widehat{\phi}|=\sqrt{\overline{\widehat{\phi}}} \hat{\phi}$ is also infinitely differentiable at 0 , and so $|\widehat{\phi}(\xi)|=\frac{1}{\sqrt{2 \pi}}+O\left(|\xi|^{\alpha}\right)$ with $\alpha$ an integer and $\sigma=\alpha / 2+1 / 2$ (note $d=1$ here). However $\alpha$ must be even since $|\widehat{\phi}(\xi)|$ always has a maximum of $\frac{1}{\sqrt{2 \pi}}$ at $\xi=0$.

In such cases the Strang-Fix conditions, which indicate integer convergence orders and are related to moment and polynomial representation conditions, are entirely equivalent to those above. However, for non-compactly supported scaling functions supported cases the two theories can diverge, in particular our results allow for non-integer optimal convergence rates (see [KR1]).

The proofs for the new results 5-9 above are given in section 7. These hold for multiresolution, scaling, and wavelet expansions when they are defined.

\section{Preliminaries for proofs}

Let $P_{n}$ and $Q_{n}$ be the kernels of the $L^{2}$ projections onto the spaces $V_{n}$ and $W_{n}$, respectively. We inverse Fourier transform and obtain

$$
\widehat{P}_{n}(x,-\xi)=\mathcal{F}_{y}^{-1} P_{n}(x, y) ; \quad \widehat{Q}_{n}(x,-\xi)=\mathcal{F}_{y}^{-1} Q_{n}(x, y)
$$

with

$$
\widehat{P}_{n}(x,-\xi)=(2 \pi)^{-d / 2} \int P_{n}(x, y) e^{i \xi y} d y
$$

and $\widehat{Q}_{n}(x,-\xi)$ defined similarly. The transforms converge everywhere and are continuous in $\xi$ if $|P(x, y)| \leq H(x-y)$ with $H \in[\mathrm{RB}]$ (see Def. 1.4). As usual, we have defined $P=P_{0}$ here. The same conclusions hold if $\psi(x) \ln (2+|x|) \in[\mathrm{RB}]$ [KKR1].

The error $E_{n} \equiv I-P_{n}$ is bounded in $L^{2}$. In Fourier space its kernel is [KR1] 


$$
\left(E_{n} f\right)(x)=\tilde{E}_{n} \mathcal{F} f,
$$

where $\widetilde{E_{\mathrm{n}}}$ has the kernel (in the variable $\xi$ )

$$
\widetilde{E}_{n}(x, \xi)=(2 \pi)^{-d / 2} e^{i x \xi}-\widehat{P}_{n}(x,-\xi),
$$

We denote $E=E_{0}$.

Recall the scaling property

$$
P_{n}(x, y)=2^{n d} P_{0}\left(2^{n} x, 2^{n} y\right),
$$

which implies

$$
\widetilde{E_{n}}(x, \xi)=\widetilde{E}\left(2^{n} x, 2^{-n} \xi\right) .
$$

Also under our assumptions on the scaling function $\phi$, the Fourier kernel $\widetilde{E}(x, \xi)$ of the remainder operator $E$ is

$$
\widetilde{E}(x, \xi)=(2 \pi)^{-d / 2} e^{i x \xi}-Z(x,-\xi) \overline{\widehat{\phi}}(\xi),
$$

where

$$
Z(x, \xi) \equiv \sum_{k} \phi(x-k) e^{-i \xi k}=\sum_{k} \phi(x+k) e^{i \xi k}
$$

is the Zak transform of $\phi$.

For later reference, it follows from [KR1], equation (3.12) and its sequel, along with properties of the Zak transform, the Poisson summation formula, and the scaling function $\phi$, that the Zak transform can be written

$$
\begin{aligned}
Z(x, \xi) & =e^{-i \xi x} \sum_{l \in \mathbb{Z}^{d}}(2 \pi)^{d / 2} \hat{\phi}(2 \pi l-\xi) e^{2 \pi i x l} \\
& =(2 \pi)^{d / 2} e^{-i \xi x} \sum_{\epsilon \in F} m_{0}(\pi \epsilon-\xi / 2) \sum_{l \in 2 \mathbb{Z}^{d}+\epsilon} \hat{\phi}(\pi l-\xi / 2) e^{2 \pi i x l},
\end{aligned}
$$

where $F=\{0,1\}^{d}$.

In addition, as calculated in [KR1], we have from (2.6)

$$
\begin{aligned}
\tilde{E}(x, \xi) & =(2 \pi)^{-d / 2} e^{i x \xi}-Z(x,-\xi) \overline{\hat{\phi}}(\xi) \\
& =(2 \pi)^{-d / 2} e^{i x \xi}\left(1-\sum_{l \in \mathbb{Z}^{d}}(2 \pi)^{d} \hat{\phi}(2 \pi l+\xi) e^{2 \pi i x l} \overline{\hat{\phi}}(\xi)\right) .
\end{aligned}
$$

The second factor can be written in the form 


$$
\begin{aligned}
1-\sum_{l \in \mathbb{Z}^{d}}(2 \pi)^{d} & \hat{\phi}(2 \pi l+\xi) e^{2 \pi i x l} \overline{\hat{\phi}}(\xi) \\
= & \left(1-(2 \pi)^{d}|\hat{\hat{\phi}}(\xi)|^{2}\right)-\sum_{l \neq 0}(2 \pi)^{d} \hat{\phi}(\xi) \hat{\phi}(2 \pi l+\xi) e^{2 \pi i x l} .
\end{aligned}
$$

For completeness we state a proposition relating approximation orders and operator norms, and two propositions relating operator norms and kernels.

Proposition 2.1 [KR1]: Assume a Banach space $A$, a normed linear space $B$, and a sequence of bounded operators $Q_{n}: A \rightarrow B$. Then the sequence $Q_{n}$ has order of approximation $\beta(n)$, i.e., $\left\|\left(I-Q_{n}\right) f\right\|_{B} \leq C_{f} \beta(n)$ for all $f \in A$, if and only if the operator norm $\left\|I-Q_{n}\right\| \leq C^{\prime} \beta(n)$, where $C^{\prime}, C_{f}$ are constants (the latter depending on f).

Proposition 2.2: An operator $R: H^{\mathrm{s}} \rightarrow L^{\infty}$ with kernel $\widetilde{R}(x, \xi)$ defined by

$$
R f(x) \equiv \int \widetilde{R}(x, \xi) \widehat{f}(\xi) d \xi
$$

has operator norm

$$
\|R\|_{H^{s} \rightarrow L^{\infty}}^{2}=\sup _{x} \int|\widetilde{R}(x, \xi)|^{2}\left(1+|\xi|^{2}\right)^{-s} d \xi
$$

Proposition 2.3 [KR1]: For $s \in \mathbb{R}$, the operator $R: H_{h}^{s} \rightarrow L^{\infty}$ defined by equation $(2.11)$ is bounded if and only if the kernel $\widetilde{R}(x, \xi)$ satisfies

$$
\int|\widetilde{R}(x, \xi)|^{2}|\xi|^{-2 s} d \xi<C<\infty
$$

Replacing the operator $R$ by $E=I-P$ we get:

Corollary 2.4: For $s>d / 2$ the $M R A\left\{P_{n}\right\}$ has best pointwise order of approximation $s-d / 2$ in $H^{\mathrm{s}}$ if and only if $\widetilde{E}(x, \xi) \in \mathcal{F} H_{h}^{-s}$ in the variable $\xi$, uniformly in $x$, i.e., iff $\int|\widetilde{E}(x, \xi)|^{2}|\xi|^{-2 s} d \xi$ is essentially bounded in $x$.

Proof: This follows from equivalence of $\left(a^{\prime}\right)$ and $(b)$ of Theorem 1 and Proposition 2.3.

\section{Growth rates of functions}

The following results on growth of functions are required in our proofs of sharpness of the best Sobolev parameter $\sigma$, and our main result, Theorem 5. The proofs are available for reference in an appendix to this paper on the Internet at http://math.bu.edu/people/mkon/, with the same title as this paper. 
Definitions 3.1: A function $f(x)$ on an open set $\mathcal{O}$ is locally bounded if it is bounded on compact sets. We denote by $B^{d}$ the unit ball of $\mathbb{R}^{d}$.

Lemma 3.2: Given a locally bounded positive function $A(x)$ on $B^{d}-\{0\}$ and $\alpha \neq 0$, (a) We have $A(x)=O\left(|x|^{-\alpha}\right)$ if and only if

$$
|A(x)-A(x / 2)|=O\left(|x|^{-\alpha}\right)
$$

where if $\alpha<0$ we assume $A(0)=\lim _{x \rightarrow 0} A(x)=0$.

(b) If $\alpha<0$ and $A(0)=\lim _{x \rightarrow 0} A(x)$ exists, but we do not assume $A(0)=0$, then in statement

(a), $A(x)=O\left(|x|^{-\alpha}\right)$ is replaced by $|A(x)-A(0)|=O\left(|x|^{-\alpha}\right)$.

(c) In (a), $O(\cdot)$ may be replaced by $o(\cdot)($ as $x \rightarrow 0)$.

Definitions 3.3: Two functions $\alpha(\cdot)$ and $\beta(\cdot)$ are equivalent, $\alpha(\cdot) \sim \beta(\cdot)$, if there exist positive constants $\mathrm{c}_{1}$ and $\mathrm{c}_{2}$ such that for every $f$ in their domain,

$$
\mathrm{c}_{1} \alpha(f) \leq \beta(f) \leq \mathrm{c}_{2} \alpha(f)
$$

For $C>1$, we define $\mathcal{L}_{C}=\mathcal{L}_{C}\left(\mathbb{R}^{d}\right)$ to be the class of positive radial functions $g(|x|)$ on $\mathbb{R}^{d}$ satisfying $g(b \rho) \sim g(\rho)$ for $\rho>0$ and $1 \leq b \leq 2$, i.e., such that for all $\rho>0$,

$$
1 / C \leq \frac{g(b \rho)}{g(\rho)} \leq C .
$$

We also define the norm

$$
\|g\|_{\mathcal{L}} \equiv \int d x|x|^{-d} g(|x|)
$$

Henceforth we assume all statements involving the order parameter $c$ hold for $0<c<1$.

Theorem 3.4: The following statements are equivalent for any fixed $\alpha>0$ and a positive function $f(x)$ on the unit ball $B^{d}$ of $\mathbb{R}^{d}$, with $0<c<1$ (all integrals are restricted to the unit ball):

(a) The integral

$$
\int_{|x|>c} f(x) d x=O\left(c^{-\alpha}\right) .
$$

(b) For some (or all) $\beta<\alpha$,

$$
\int_{|x|>c} f(x)|x|^{\beta} d x=O\left(c^{\beta-\alpha}\right)
$$

[and if $\left.\beta>\alpha, \int f(x)|x|^{\beta} d x<\infty\right]$.

(b') For some (or all) $\beta \in \mathbb{R}$, 


$$
\int_{c / 2<|x|<c} f(x)|x|^{\beta} d x=O\left(c^{\beta-\alpha}\right) .
$$

(b") For some (or all) $\beta>\alpha$, and some (or all) $\gamma$ with $\gamma+\beta-\alpha<0$,

$$
\int f(x)|x|^{\beta}(c+|x|)^{\gamma} d x=O\left(c^{\gamma+\beta-\alpha}\right)
$$

[and if $\beta>\alpha$ and $\gamma+\beta-\alpha>0$, then $\lim _{c \rightarrow 0} \int f(x)|x|^{\beta}(c+|x|)^{\gamma} d x$ exists and is finite].

(c) For any function $g(|x|) \in \mathcal{L}_{C}$ such that

$$
\int|x|^{-d} g(|x|) d x<\infty
$$

it follows that

$$
\int g(|x|)|x|^{\alpha} f(x) d x<\infty
$$

for some (or all) $C>1$.

Statements in brackets [ $[\cdot]$ may be included or excluded without changing the equivalences.

In addition, $O(\cdot)$ may be replaced by $o(\cdot)$ simultaneously in all of the above statements excluding $(c)$, and the equivalences of $(a)-\left(b^{\prime \prime}\right)$ (i.e. all statements excluding $(c)$ ) continue to hold.

Remark: For completeness (though this will not be used in the paper) we remark that the conditions in the above Theorem are also equivalent to the following conditions, listed below:

$\left(b^{\prime \prime \prime}\right)$ For some (or all) $\beta>\alpha, \int_{|x|<c} f(x)|x|^{\beta} d x=O\left(c^{\beta-\alpha}\right)$.

$\left(b^{\prime \prime \prime \prime}\right)$ For some (or all) $\beta, \gamma \in \mathbb{R}, \int_{c / 2<|x|<c} f(x)|x|^{\beta}(c+|x|)^{\gamma} d x=O\left(c^{\gamma+\beta-\alpha}\right)$.

(d) $\int_{c / 2<|x|<c} f(x) d x=O\left(c^{-\alpha}\right)$.

(e) $\int_{c / 2<|x|<c}|x|^{\alpha} f(x) d x=O(1)$.

We now state a corollary which gives uniformity for Theorem 3.4.

Corollary 3.5: Let $\left\{f_{q}\right\}_{q \in Q}$ be a family of positive functions from $B^{d}$ to $\mathbb{R}$. The following statements (with all inequalities uniform in q) are equivalent for fixed $\alpha>0$ (note all integrals below are restricted to $B^{d}$ ) and $0<c<1$ :

(a) The integral

$$
\int_{|x|>c} f_{q}(x) d x \leq K_{1} c^{-\alpha} .
$$

(b) For some (or all) $\beta<\alpha$, 


$$
\int_{|x|>c} f_{q}(x)|x|^{\beta} d x \leq K_{2} c^{\beta-\alpha}
$$

[and for $\beta>\alpha, \int f_{q}(x)|x|^{\beta} d x<K$ for some $K$ independent of $\left.q\right]$.

$\left(b^{\prime}\right)$ For some (or all) $\beta \in \mathbb{R}$,

$$
\int_{c / 2<|x|<c} f_{q}(x)|x|^{\beta} d x \leq K_{3} c^{\beta-\alpha} .
$$

$\left(b^{\prime \prime}\right)$ For some (or all) choices of $\beta$ with $\beta>\alpha$ and $\gamma$ with $\gamma+\beta-\alpha<0$,

$$
\int f_{q}(x)|x|^{\beta}(c+|x|)^{\gamma} d x \leq K_{4} c^{\gamma+\beta-\alpha}
$$

[gand if $\beta>\alpha$ and $\gamma$ satisfy $\gamma+\beta-\alpha>0$, then $\int f_{q}(x)|x|^{\beta}(c+|x|)^{\gamma} d x \leq K$ for some $K$ independent of $q]$.

(c) For any function $g(|x|) \in \mathcal{L}_{C}$ such that $\|g\|_{\mathcal{L}} \equiv \int|x|^{-d} g(|x|) d x<\infty$, it follows that

$$
\int g(|x|)|x|^{\alpha} f_{q}(x) d x<K_{5}\|g\|_{\mathcal{L}},
$$

for some (or all) $C>1$.

$\left(c^{\prime}\right)$ For any function $g(|x|) \in \mathcal{L}_{C}$ such that $\|g\|_{\mathcal{L}}<\infty$, it follows that

$$
\int g(|x|)|x|^{\alpha} f_{q}(x) d x<K(g),
$$

for some (or all) $C>1$, where $K(g)$ depends on g but not on $q$.

The above constants $K_{i}$ are all equivalent, i.e., there is a constant $\mu$ such that $\frac{1}{\mu} K_{1} \leq K_{2}, K_{3}, \ldots, K_{5} \leq \mu K_{1}$, for any fixed choice of $\alpha, \beta$, and $\gamma$. The bracketed statements in (b) and $\left(b^{\prime \prime}\right)$ can be included or excluded without changing the equivalences.

Remark: For completeness (though this will not be used in this paper), we remark that the conditions of Corollary 3.5 are also equivalent to the following:

$\left(b^{\prime \prime \prime}\right)$ For some (or all) $\beta>\alpha, \int_{|x|<c} f_{q}(x)|x|^{\beta} d x \leq K_{6} c^{\beta-\alpha}$.

$\left(b^{\prime \prime \prime \prime}\right)$ For some (or all) $\beta, \gamma \in \mathbb{R}, \int_{c / 2<|x|<c} f_{q}(x)|x|^{\beta}(c+|x|)^{\gamma} d x \leq K_{7} c^{\gamma+\beta-\alpha}$.

(d) $\int_{c / 2<|x|<c} f_{q}(x) d x \leq K_{8} c^{-\alpha}$.

(e) $\int_{c / 2<|x|<c}|x|^{\alpha} f_{q}(x) d x \leq K_{9}$.

with the constants $K_{6}$ through $K_{9}$ equivalent to $K_{1}$ through $K_{5}$.

The next Corollary relates divergence rates of two integrals as $c \longrightarrow 0$ : 
Corollary 3.6: Let $\left\{f_{q}^{*}\right\}_{q \in Q}$ denote a family of positive functions $f_{q}^{*}: B^{d} \rightarrow \mathbb{R}$. The following statements (with all inequalities uniform in q) are equivalent for given $\gamma_{1}, \delta, \eta \in \mathbb{R}$, with $\eta<-\gamma_{1}$, and $\eta, \eta-\delta$ positive.

(a) $\int_{B^{d}} f_{q}^{*}(x)(c+|x|)^{\gamma_{1}} d x \leq K_{1} c^{-\eta}$, for some $K_{1}>0$ and all $0<c<1$.

(b) $\int_{B^{d}} f_{q}^{*}(x)(c+|x|)^{\gamma_{1}+\delta} d x \leq K_{2} c^{-\eta+\delta}$, for some $K_{2}>0$ and all $0<c<1$.

Furthermore, if the above assumptions hold except that $\eta-\delta$ is negative, then (a) implies that the left side of $(b)$ is bounded uniformly in $q$.

Proof of Corollary 3.6: We first prove $(a) \Leftrightarrow(b)$ under the initial assumptions. Note that for fixed $\alpha$, statement $\left(b^{\prime \prime}\right)$ of Corollary 3.5 is equivalent to itself if "for some" is replaced by "for all". By the symmetry of $(a)$ and $(b)$, it suffices to prove $(a) \Rightarrow(b)$. Thus assume (a) holds. We define constants $\alpha>0, \beta, \gamma$ which satisfy

$$
\gamma=\gamma_{1} \text { and } \gamma+\beta-\alpha=-\eta \text {. }
$$

Note that since $\eta<-\gamma_{1}$ it follows $\beta>\alpha$. Defining the function $f_{q}$ by

$$
f_{q}^{*}(x) \equiv f_{q}(x)|x|^{\beta}
$$

we see that $\left(b^{\prime \prime}\right)$ of Corollary 3.5 is satisfied for our choice of $\alpha, \beta, \gamma$. Let $\delta<\eta$, and now replace $\gamma_{1}$ by $\gamma_{1}+\delta$, and replace $\eta$ by $\eta-\delta$. With these new values of $\gamma_{1}$ and $\eta$, we keep $\alpha$ and $\beta$ unchanged, so that (3.2) is still satisfied. By the equivalence of the "for some" and "for all" versions of statement $\left(b^{\prime \prime}\right)$ in Corollary 3.5, it follows that for this new value of $\gamma$, $\left(b^{\prime \prime}\right)$ still holds. However, $\left(b^{\prime \prime}\right)$ of Corollary 3.5 with the new value of $\gamma$ is the same as $(b)$ of this Corollary, proving $(b)$ as desired.

Now consider the case where $\eta-\delta$ is negative. We will show that $(a)$ implies that the left side of $(b)$ is uniformly bounded. We maintain all of the original assumptions of this Corollary, with the only change that now $\eta-\delta$ is assumed negative instead of positive. Again define $\alpha>0, \beta, \gamma$ so that (3.2) holds. Then with these values of $\alpha, \beta, \gamma,(a)$ above is again equivalent to the unbracketed part of $\left(b^{\prime \prime}\right)$ of Corollary 3.5.

Since the unbracketed part of $\left(b^{\prime \prime}\right)$ for one value of $\gamma$ implies the bracketed part for all values of $\gamma$ such that $\gamma+\beta-\alpha>0$, it follows that the bracketed part of Corollary 3.5 holds for the new value of $\gamma$. Thus

$$
\int_{B^{d}} f_{q}(x)|x|^{\beta}(c+|x|)^{\gamma_{1}+\delta} d x \leq K
$$

for some $K>0$. This completes the proof.

\section{Convergence rates and the best Sobolev parameter}

The main result of this section, Theorem 4.3, shows that for any wavelet expansion the best pointwise rate of convergence in $H^{s}$ is independent of $s$ for $s>\sigma$, where $\sigma$ is the best Sobolev parameter.

We recall that $E_{n}=I-P_{n}$, and $Q_{n}=E_{n}-E_{n+1}$; see (2.1) and (2.3) for definitions of $\widehat{Q}_{n}$ and $\widetilde{E}_{n}$. 
The next proposition will be used later to establish that the function $K_{s}(c)$ can replace $I_{s}(c)$ (see Def. 1.6) in the statements of our theorems.

Proposition 4.1: If $s>d / 2, \alpha \neq 2 s-d$, and $\alpha>0$, then for $0<c<1$

$$
M(c) \equiv\left\|\int_{|\xi|>c}\left|\widetilde{E_{n}}(x, \xi)\right|^{2}|\xi|^{-2 s} d \xi\right\|_{\infty}=O\left(c^{-\alpha}\right)
$$

if and only if

$$
N(c) \equiv\left\|\int_{|\xi|>c}\left|\widehat{Q}_{n}(x, \xi)\right|^{2}|\xi|^{-2 s} d \xi\right\|_{\infty}=O\left(c^{-\alpha}\right),
$$

where the norms are in $x$.

Proof: Assume $M(c)=O\left(c^{-\alpha}\right)$. Using scaling properties of the kernels (2.5),

$$
\begin{aligned}
N(c) & =\left\|\int_{|\xi|>c}\left|\widehat{Q}_{n}(x, \xi)\right|^{2}|\xi|^{-2 s} d \xi\right\|_{\infty} \\
& =\left\|\int_{|\xi|>c}\left|\widetilde{E_{n}}(2 x, \xi / 2)-\widetilde{E}_{n}(x, \xi)\right|^{2}|\xi|^{-2 s} d \xi\right\|_{\infty} \\
& \leq K\left(\left\|\int_{|\xi|>c}\left|\widetilde{E_{n}}(2 x, \xi / 2)\right|^{2}|\xi|^{-2 s} d \xi\right\|_{\infty}+\left\|\int_{|\xi|>c}\left|\widetilde{E_{n}}(x, \xi)\right|^{2}|\xi|^{-2 s} d \xi\right\|_{\infty}\right) \\
& =K\left(2^{-2 s+d} M(c / 2)+M(c)\right),
\end{aligned}
$$

proving $N(c)=O\left(c^{-\alpha}\right)$.

Conversely assume $N(c)=O\left(c^{-\alpha}\right)$. Then we have

$$
\begin{aligned}
N(c) & =\left\|\int_{|\xi|>c} d \xi\left|\widetilde{E_{n}}(2 x, \xi / 2)-\widetilde{E_{n}}(x, \xi)\right|^{2}|\xi|^{-2 s}\right\|_{\infty} \\
& \geq\left(\left\|\int_{|\xi|>c} d \xi|\xi|^{-2 s}\left|\widetilde{E_{n}}(2 x, \xi / 2)\right|^{2}\right\|_{\infty}^{1 / 2}-\left\|\int_{|\xi|>c} d \xi|\xi|^{-2 s}\left|\widetilde{E_{n}}(x, \xi)\right|^{2}\right\|_{\infty}^{1 / 2}\right)^{2} \\
& =\left(2^{d / 2-s}\left\|\int_{|\xi|>c / 2} d \xi|\xi|^{-2 s}\left|\widetilde{E_{n}}(x, \xi)\right|^{2}\right\|_{\infty}^{1 / 2}-\left\|\int_{|\xi|>c} d \xi|\xi|^{-2 \mathrm{~s}}\left|\widetilde{E_{n}}(x, \xi)\right|^{2}\right\|_{\infty}^{1 / 2}\right)^{2} \\
& =\left(2^{d / 2-s} M(c / 2)^{1 / 2}-M(c)^{1 / 2}\right)^{2},
\end{aligned}
$$

where all $L^{\infty}$ norms are in $x$.

To show this implies $M(c)=O\left(c^{-\alpha}\right)$, define $S(c)=c^{s-d / 2} M(c)^{1 / 2}$. Then

$$
N(c)=\left(2^{d / 2-s} S(c / 2)(c / 2)^{d / 2-s}-c^{d / 2-s} S(c)\right)^{2}=c^{d-2 s}(S(c / 2)-S(c))^{2} .
$$

Since $N(c)=O\left(c^{-\alpha}\right)$

$$
|S(c / 2)-S(c)|=O\left(c^{-\alpha / 2+s-d / 2}\right)
$$


By (a) of Lemma 3.2, if $\alpha>2 s-d$, we have $S(c)=O\left(c^{-\alpha / 2+s-d / 2}\right)$, and so $M(c)=O\left(c^{-\alpha}\right)$ as desired.

If on the other hand $\alpha<2 s-d$, in order to apply Lemma 3.2 (a) we show $S(0) \equiv \lim _{c \rightarrow 0}$ $S(c)=0$. To this end, we first bound $M(c)$ as follows. Define

$$
Y(\xi)=\|\widetilde{E}(x, \xi)\|_{\infty}=\|(2 \pi)^{-d / 2} e^{i x \xi}-Z\left(x,-\xi \overline{\widehat{\phi}}(\xi) \|_{\infty}\right.
$$

where the above norms are in $x$. It is not difficult to show (see [KR1] and the remarks for $F(\xi)$ before equation (2.3.16) there) that $Y(\xi) \underset{\xi \rightarrow 0}{\longrightarrow} 0$.

Note also that

$$
\begin{aligned}
\int_{c / 2<|\xi|<c}\|\widetilde{E}(x, \xi)\|_{\infty, x}^{2}|\xi|^{-2 s} d \xi & =\int_{c / 2<|\xi|<c} Y(\xi)^{2}|\xi|^{-2 s} d \xi \\
& \leq K_{1} c^{-2 s} \int_{c / 2<|\xi|<c} Y(\xi)^{2} d \xi \\
& \leq K_{2} c^{-2 s} c^{d} \sup _{|\xi|<c} Y(\xi)^{2} \\
& =o\left(c^{d-2 s}\right) .
\end{aligned}
$$

Thus

$$
M(c) \leq \int_{|\xi|>c}\left\|\widetilde{E_{n}}(x, \xi)\right\|_{\infty, x}^{2}|\xi|^{-2 s} d \xi=o\left(c^{d-2 s}\right),
$$

using the equivalence of (b) and (b') (in the case where $O$ is replaced by $o$ ) in Theorem 3.4, since by our assumptions $d-2 s<0$. Thus

$$
\lim _{c \rightarrow 0} S(c)=\lim _{c \rightarrow 0} c^{s-d / 2} M(c)^{1 / 2}=o(1) \quad(c \rightarrow 0),
$$

so that $S(0)=0$.

Now applying Lemma 3.2 (a), we have by (4.1) in the case $0<\alpha<2 s-d$ that $S(c)=O\left(c^{-\alpha / 2+s-d / 2}\right)$, so $M(c)=O\left(c^{-\alpha}\right)$ in this case as well, completing the proof.

Theorem 4.2: For $s \geq 0$, the $M R A\left\{P_{n}\right\}$ has pointwise order of convergence $r \in \mathbb{R}$ in $H^{s}$ if and only if

$$
\left.\int \tilde{E}(x, \xi)\right|^{2}(c+|\xi|)^{-2 s} d \xi \leq K c^{2(r-s)+d}
$$

for $0<c<1$, uniformly in $x$.

Proof: Assume first (4.2) holds. Then for $f \in H^{s}$ (letting $c=2^{-n}$ ) 


$$
\begin{aligned}
\left|E_{n} f(x)\right|^{2} & =\left|\int \widetilde{E_{n}}(x, \xi) \hat{f}(\xi) d \xi\right|^{2} \\
& \leq \int\left|\widetilde{E_{n}}(x, \xi)\right|^{2}(1+|\xi|)^{-2 s} d \xi \int|\hat{f}(\xi)|^{2}(1+|\xi|)^{2 s} d \xi \\
& =c^{2 s-d} \int|\widetilde{E}(x / c, \xi)|^{2}(c+|\xi|)^{-2 s} d \xi \int|\hat{f}(\xi)|^{2}(1+|\xi|)^{2 s} d \xi \\
& \leq K c^{2 s-d-2 s+2 r+d} \\
& =K 2^{-2 n r},
\end{aligned}
$$

independently of $x$. In the third line we used the scaling property (2.5) for kernels.

Conversely assume the MRA has approximation order $r$ in $\mathrm{H}^{s}$. Then for $\mathrm{f} \in H^{s}$ we have $\left\|E_{n} f\right\|_{\infty} \leq K 2^{-n r}=K c^{r}$. Thus by Proposition $2.1\left\|E_{n}\right\|_{H^{s} \rightarrow L^{\infty}}=O\left(2^{-n r}\right)$, implying by Proposition 2.2 (and the equivalence of the factors $(1+|\xi|)^{-2 s}$ and $\left.\left(1+|\xi|^{2}\right)^{-s}\right)$ that

$$
\begin{aligned}
K 2^{-2 n r} & =K c^{2 r} \\
& \geq \operatorname{ess} \sup _{x} \int\left|\widetilde{E_{\mathrm{n}}}(x, \xi)\right|^{2}(1+|\xi|)^{-2 s} \mathrm{~d} \xi \\
& =c^{-d+2 s} \operatorname{ess} \sup _{x} \int|\widetilde{E}(x, \xi)|^{2}(c+|\xi|)^{-2 s} d \xi,
\end{aligned}
$$

implying

$$
\operatorname{ess} \sup _{x} \int|\widetilde{E}(x, \xi)|^{2}(c+|\xi|)^{-2 s} d \xi \leq K c^{2(r-s)+d}
$$

as desired.

Theorem 4.3: If the best Sobolev parameter $\sigma \neq 0$, then the best pointwise order of convergence of the MRA $\left\{P_{n}\right\}$ in $H^{s}$ is independent of $s$ for $s>\sigma$.

Proof: Assume we have approximation order $r$ in $H^{s}$. Then uniformly a.e. in $x$, referring to the definition of $\widetilde{E}(x, \xi)$ in $(2.3)$ and Theorem 4.2 ,

$$
\int|\tilde{E}(x, \xi)|^{2}(c+|\xi|)^{-2 s} d \xi \leq K c^{-2 s+2 r+d}
$$

Assume initially that $s>\sigma+1 / 2$. We apply Corollary 3.6 with $f_{x}^{*}(\xi)=|\widetilde{E}(x, \xi)|^{2}$ and $\gamma_{1}=-2 s, \eta=2 s-2 r-d$, and $\delta=1$. We show the hypotheses and (a) of the corollary are satisfied as follows. First, $\eta+\gamma_{1}=-2 r-d<0$. Second,

$$
\int f_{x}^{*}(\xi)(c+|\xi|)^{\gamma_{1}} d \xi=\int|\tilde{E}(x, \xi)|^{2}(c+|\xi|)^{-2 s} d \xi \leq K_{1} c^{2 r-2 s+d}=K_{1} c^{-\eta}
$$

Note that since $s>\sigma+1 / 2$, it follows that $(I-P): H_{h}^{s} \rightarrow L^{\infty}$ is unbounded. The integral on the left side of (4.4) diverges as $c \rightarrow 0$, since by the equivalence of $\left(a^{\prime}\right)$ and $(b)$ 
in Theorem 1 and Corollary 2.4, $\int|\tilde{E}(x, \xi)|^{2}|\xi|^{-2 s} d \xi$ is unbounded in $x$ for $s>\sigma$. Thus $\eta>0$.

Now, for an $\epsilon>0$ (to be determined later), we claim that (using $\delta=1$ ), uniformly a.e. in $x$,

$$
\int|\tilde{E}(x, \xi)|^{2}(c+|\xi|)^{-2(s-(1 / 2+\epsilon))} d \xi \leq K c^{-2(s-(1 / 2+\epsilon))+2 r+d}=K c^{1-\eta+2 \epsilon}
$$

We first define $\epsilon$ more precisely. We will require that $\epsilon$ be sufficiently small that

$$
s-(1 / 2+\epsilon)>\sigma \text {. }
$$

Further, we require that $\epsilon$ be chosen so that the exponent $1-\eta+2 \epsilon$ in (4.5) is nonzero.

Before continuing we will show that the part of the integral in (4.5) which is outside the unit ball remains uniformly bounded a.e. in $x$ and $c$. To this end, note that the exponent in the integral satisfies

$$
-2(s-(1 / 2+\epsilon))<-2 \sigma<-d,
$$

since $\sigma>d / 2$. Thus the integral over the outside of the ball remains uniformly bounded, since $\tilde{E}(x, \xi)$ is uniformly bounded (this follows from the definition of $E$ and from the fact that $P(x, y)$ is in all cases bounded by an $L^{1}$ convolution kernel $H(x-y)$ with $H$ radially bounded; see [KR1]).

Now we will show that under the above assumptions in fact $1-\eta+2 \epsilon<0$. Indeed, Corollary 3.6 implies that if $1-\eta+2 \epsilon>0$, then the left side of (4.5) is uniformly bounded a.e. in $x$ as $c$ varies in $(0,1)$ (this includes the portion of the integral outside the ball by the above remark). However, by (4.6), the left side of (4.5) diverges as $c \rightarrow 0$ since (as above) by Theorem 1 and Corollary 2.4, $\int|\tilde{E}(x, \xi)|^{2}|\xi|^{-2 s} d \xi$ is unbounded in $x$ for $s>\sigma$. Hence $1-\eta+2 \epsilon<0$.

Thus by Corollary 3.6, (4.5) holds, and by Theorem 4.2 we conclude that we have order of convergence $r$ in $H^{s-(1 / 2+\epsilon)}$. Thus since $s-1 / 2>s-(1 / 2+\epsilon)$, we also have order of convergence $r$ in $H^{s-1 / 2}$ (when $s>\sigma+1 / 2$ ).

Thus if $s>\sigma+1 / 2$, and we have order of approximation $r$ in $\mathrm{H}^{s}$, then we also have order of approximation $r$ in $H^{s-1 / 2}$. This means that if $S_{s}$ is the set of orders of convergence in $H^{s}$, then $S_{s-1 / 2} \supseteq S_{s}$ for $s>\sigma+1 / 2$. We know also that any order $r$ of convergence in $H^{s}$ also applies to $H^{s^{\prime}}$ for $s^{\prime}>s$, and so $S_{s}$ as a set is nondecreasing with $s$. Thus $S_{s-1 / 2} \subseteq S_{s}$. Combining the above inclusions, $S_{s-1 / 2}=S_{s}$, i.e., as a function of $s$ the set $S_{s}$ is periodic with period $1 / 2$. Combining this with the fact that $S_{s}$ is nondecreasing, we conclude the set $S_{s}$ must be constant as a function of $s$ for $s>\sigma$. Thus the valid orders of convergence are the same in $H^{s}$ for $s>\sigma$. We remark that above any positive constant could have been used in place of $1 / 2$ in the term $\sigma+1 / 2$.

Proposition 4.4: If $E: H_{h}^{\sigma} \rightarrow L^{\infty}$ is bounded, then for all $s \geq \sigma$, the $M R A\left\{P_{\mathrm{n}}\right\}$ has best order of convergence $\sigma-d / 2$ in $H^{s}$.

Proof: Our assumption on the boundedness of $E$ implies by Proposition 1.8 that $\sigma>d / 2$. 
By Theorem 1 if $0<r \leq \sigma-d / 2$, the MRA has approximation order $r$ in $H^{\sigma}$, and hence in $H^{s} \subset H^{\sigma}$ for $s>\sigma$. Thus the best approximation order is at least $\sigma-d / 2$ in $H^{s}$, and so $S_{\sigma}=[0, \sigma-d / 2]$, where $S_{s}$ denotes the set of approximation orders in $H^{s}$. Note we have used the equivalence of $(a)$ and $\left(a^{\prime}\right)$ in Theorem 1, which implies that if we have approximation order $\sigma-\mathrm{d} / 2$ in $H^{\sigma}$ this order is the largest possible in $H^{\sigma}$.

By Theorem $4.3 S_{s}$ is independent of $s>\sigma$. We claim for $s>\sigma, S_{s}=[0, \sigma-d / 2]$. First note since $S_{s}$ is increasing with $s$ and $S_{\sigma}=[0, \sigma-d / 2]$, we have $S_{s} \supseteq[0, \sigma-d / 2]$ for $s>\sigma$.

We show $S_{s} \subseteq[0, \sigma-d / 2]$ as follows. If it were true that $r>\sigma-d / 2$ and $r \in S_{s}$ then Theorem 4.3 would imply that $r \in S_{r+d / 2}$, since $r+d / 2>\sigma$. This would give by the equivalence of $\left(a^{\prime}\right)$ and $(b)$ in Theorem 1 that $I-P_{n}: H^{r+d / 2} \rightarrow L^{\infty}$ is bounded. By definition of $\sigma$ (Definitions 1.7), this would imply $\sigma \geq r+d / 2$, giving the desired contradiction. Thus it is impossible that $r \in S_{s}$ if $r>\sigma-d / 2$ and $s>\sigma$. Therefore $S_{s}=$ $[0, \sigma-d / 2]$ for $s>\sigma$ as claimed, completing the proof.

\section{Conditions for convergence rates}

With Corollary 2.4 as motivation, we define (for $0 \leq c \leq 1$ )

$$
J_{r}(c)=\sup _{x} \int_{|\xi| \geq c}|\widetilde{E}(x, \xi)|^{2}|\xi|^{-2 r} \mathrm{~d} \xi,
$$

where the sup as usual is a.e.

The following theorem is the analog of Theorem 5, using the $J$ instead of the $I$ integrals as criteria for approximation orders.

Theorem 5.1: Given an $\operatorname{MRA}\left\{P_{n}\right\}$ with $\sigma \neq 0$ :

(i) If $d / 2<s<\sigma$ the best order of approximation of $\left\{P_{\mathrm{n}}\right\}$ in $H^{\mathrm{s}}$ is $s-d / 2$.

(ii) If $s=\sigma$, the best order of approximation in $H^{s}$ is $r=\left\{\begin{array}{c}\sigma-d / 2 \text { if } J_{\sigma}(0)<\infty \\ (\sigma-d / 2)^{-} \text {if } J_{\sigma}(0)=\infty\end{array}\right.$.

(iii) If $s>\sigma$, the best order of approximation in $H^{\mathrm{s}}$ is $r=\left\{\begin{array}{l}\sigma-d / 2 \text { if } J_{\sigma+1 / 2}(c)=O(1 / c)(c \rightarrow 0) \\ \sigma-d / 2)^{-} \text {otherwise }\end{array}\right.$.

Proof: Since $\sigma \neq 0$ we have $\sigma>d / 2$ by Proposition 1.8. Statement (i) follows from the definition of $\sigma$ and from the equivalence in Theorem 1 of $\left(a^{\prime \prime}\right)$ and $(b)$. If $J_{\sigma}(0)<\infty$, statement (ii) follows from Corollary 2.4. On the other hand if $J_{\sigma}(0)=\infty$, then by Corollary 2.4, approximation order $\sigma-d / 2$ fails. However, by Proposition 1.8 the set $\Sigma$ satisfies $\Sigma=(d / 2, \sigma)$. Thus for any $s^{\prime}$ with $d / 2<s^{\prime}<\sigma$, the operator $E: H_{h}^{s^{\prime}} \rightarrow L^{\infty}$ is bounded, and so the MRA has approximation order $s^{\prime}-d / 2$ in $H^{s^{\prime}}$ and hence also in $H^{\sigma}$. Therefore, in $H^{\sigma}$ we have all orders of approximation less than $\sigma-d / 2$, which means the order of approximation is $(\sigma-d / 2)^{-}$.

It remains to prove (iii). Since by Theorem 4.3 we have best order of approximation independent of s for $\mathrm{s}>\sigma$, we need only consider a specific value of $s$, say $s=\sigma+1 / 2$, and 
find the best approximation order in $H^{\mathrm{s}}$. By Theorem 4.2 this is the supremum of values of $r$ for which

$$
\int|\widetilde{E}(x, \xi)|^{2}(c+|\xi|)^{-2 \sigma-1} \mathrm{~d} \xi \leq K c^{-2 \sigma+2 r+d-1} .
$$

for $0<c<1$. For any $r$ where (5.1) holds, we have order of convergence $r$ in $H^{\sigma+1 / 2}$.

We first show (letting $r=\sigma-d / 2$ )

$$
\int|\widetilde{E}(x, \xi)|^{2}(c+\xi \mid)^{-2 \sigma-1} \mathrm{~d} \xi \leq K c^{-2 \sigma+2(\sigma-\mathrm{d} / 2)+d-1}=K / c
$$

if

$$
J_{\sigma+1 / 2}(c)=O\left(c^{-1}\right),
$$

(recall all order statements in $c$ hold for $0<c<1$ only). Then we show that (5.2) is true for $\sigma$ replaced by $\sigma^{-}$(i.e. it holds for all $\sigma^{\prime}<\sigma$ ) if (5.3) fails. We also need to show these choices of $\sigma$ and $\sigma^{-}$are best possible (largest possible) in (5.2).

To prove the first statement, i.e., equation (5.2), assume (5.3) holds, i.e.,

$$
\sup _{x} \int_{|\xi| \geq c}|\widetilde{E}(x, \xi)|^{2}|\xi|^{-2 \sigma-1} d \xi \leq K_{1} c^{-1} \text {. }
$$

With the goal of applying Corollary 3.5, let $f_{x}(\xi)=|\widetilde{E}(x, \xi)|^{2}|\xi|^{-2 \sigma-1}$ and $\alpha=1$. Let $\beta=2 \sigma+1$ and $\gamma=-2 \sigma-1$, so that $\gamma+\beta-\alpha=-1<0$ and $\beta-\alpha=2 \sigma>0$. Applying the equivalence of $(a)$ and $\left(b^{\prime \prime}\right)$ in Corollary 3.5, we conclude

$$
\sup _{x} \int|\widetilde{E}(x, \xi)|^{2}(c+|\xi|)^{-2 \sigma-1} d \xi \leq K_{1} c^{-1} .
$$

This proves (5.2) and shows that if $J_{\sigma+1 / 2}(c)=O\left(c^{-1}\right)$ we have approximation order $\sigma-$ $d / 2$ in $H^{\sigma+1 / 2}$ by Theorem 4.2.

Note that this order is in fact best by Theorem 1. Indeed, since this order is the same in all $H^{s}$ for $s>\sigma$, it holds for $s=\sigma+\epsilon$ for $\epsilon>0$. However by Theorem 1 the approximation order in $H^{s}$ in this case cannot be better than $\sigma+\epsilon-d / 2$, and so the (constant) order of approximation in $H^{s}$ for $s>\sigma$ cannot be better than this for all $\epsilon>0$, and hence cannot be better than $\sigma-d / 2$. Thus for $s=\sigma+1 / 2$ (and so for all $s>\sigma$ ) the best approximation order in $H^{s}$ is $\sigma-d / 2$ as desired.

Now we consider when $J_{\sigma+1 / 2}(c)=O(1 / c)$ fails to hold, and show we have best approximation order $(\sigma-d / 2)^{-}$in $H^{\sigma+1 / 2}$. By (ii) above the best approximation order is at least $(\sigma-d / 2)^{-}$and we must show it cannot be better. However since (5.3) fails by our assumption, it is easy to show by the same arguments as above that (5.2) fails. By Theorem 4.2 therefore, we fail to have order of convergence $(\sigma-d / 2)$ in $H^{\sigma+1 / 2}$, so the best approximation order must be $(\sigma-d / 2)^{-}$. Thus by Theorem 4.3 this is the best approximation order in $H^{s}$ for all $s \geq \sigma$.

\section{Preliminaries for the proof of Theorem 5}


We present some technical lemmas required in the proofs of the main results, parts (iv) and (v) of Theorem 5. Recall that the class $\mathcal{L}_{C}$ and the norm $\|\cdot\|_{\mathcal{L}}$ are given in Def. 3.3 , and that the integral

$$
I_{\sigma+1 / 2}(c) \equiv \int_{1 \geq|\xi| \geq \mathrm{c}}\left(1-(2 \pi)^{d / 2}|\hat{\phi}(\xi)|\right)|\xi|^{-2 \sigma-1} d \xi .
$$

Lemma 6.1: Let $\sigma>d / 2$ and $C>1$. Assume $I_{\sigma+1 / 2}(c)=O(1 / c)($ for $0<c<1)$. Then for $g(|\xi|) \in \mathcal{L}_{C}$ with $\|g\|_{\mathcal{L}}<\infty$, we have (defining $h(\xi)=g(|\xi|)|\xi|^{-2 \sigma}$ )

(i) $\int_{|\xi|<1}\left(1-\left|m_{0}(\xi / 2)\right|^{2}\right) h(\xi) d \xi<\infty$

(ii) $\int_{|\xi|<1}\left|m_{0}(\pi \epsilon+\xi / 2)\right|^{2} h(\xi) d \xi<\infty$ for $\epsilon \in F=\{1,0\}^{d}$.

Proof: Using the assumption $I_{\sigma+1 / 2}(c)=O(1 / c)$ and the equivalence of $(a)$ and $(c)$ in Corollary 3.5, letting $f(\xi)=\left(1-(2 \pi)^{d / 2}|\widehat{\phi}(\xi)|\right)|\xi|^{-2 \sigma-1}$ and $\alpha=1$, we have

$$
\int_{|\xi|<1}\left(1-(2 \pi)^{d / 2}|\widehat{\phi}(\xi)|\right) h(\xi) d \xi<\infty .
$$

In addition,

$$
\int_{|\xi|<1}\left(1-(2 \pi)^{d}|\widehat{\phi}(\xi)|^{2}\right) h(\xi) d \xi=\int_{|\xi|<1}\left(1-(2 \pi)^{d / 2}|\widehat{\phi}(\xi)|\right) \quad\left(1+(2 \pi)^{d / 2}|\widehat{\phi}(\xi)|\right) h(\xi) d \xi,
$$

so

$$
\int_{|\xi|<1}\left(1-(2 \pi)^{d}|\widehat{\phi}(\xi)|^{2}\right) h(\xi) d \xi<\infty
$$

Thus since

$$
\left|m_{0}(\xi)\right|^{2}-1=\left((2 \pi)^{d}|\widehat{\phi}(2 \xi)|^{2}-1\right)-\left|m_{0}(\xi)\right|^{2}\left((2 \pi)^{d}|\widehat{\phi}(\xi)|^{2}-1\right),
$$

we have

$$
\int_{|\xi|<1}\left(1-\left|m_{0}(\xi / 2)\right|^{2}\right) h(\xi) \mathrm{d} \xi<\infty
$$

proving $(i)$.

Additionally, it is known that (e.g., [KR1, equation (2.3.9) and Lemma 2.3.1]),

$$
\sum_{\epsilon \in F}\left|m_{0}(\xi+\pi \epsilon)\right|^{2}=1 ; \quad \sum_{\ell \in \mathbb{Z}^{d}}|\hat{\phi}(\xi+2 \pi \ell)|^{2}=(2 \pi)^{-d} .
$$

If $\epsilon \neq 0$, by $(6.4)$, 


$$
\left|m_{0}(\pi \epsilon+\xi / 2)\right|^{2}=1-\sum_{\epsilon^{\prime} \neq \epsilon}\left|m_{0}\left(\pi \epsilon^{\prime}+\xi / 2\right)\right|^{2} \leq 1-\left|m_{0}(\xi / 2)\right|^{2}
$$

so by $(6.3)$

$$
\int_{|\xi|<1}\left|m_{0}(\pi \epsilon+\xi / 2)\right|^{2} h(\xi) d \xi<\infty
$$

yielding $(i i)$ and completing the proof.

The Fourier kernel $\tilde{E}(x, \xi)$ is given in (2.6). We also have:

Lemma 6.2: Let $\sigma>d / 2$ and $C>1$. Assume $I_{\sigma+1 / 2}(c)=O(1 / c)(0<c<1)$. Then the kernel $\tilde{E}(x, \xi)$ satisfies

$$
\int_{|\xi|<1}\left|\|\tilde{E}(x, \xi)\|_{\infty}-\|\tilde{E}(x, \xi / 2)\|_{\infty}\right| h(\xi) d \xi<\infty
$$

for any $g(|\xi|) \in \mathcal{L}_{C}$ with $\|g\|_{\mathcal{L}}<\infty$. Here $h(\xi)=g(\xi)|\xi|^{-2 \sigma}$, and the $\|\cdot\|_{\infty}$ norm is taken with respect to $x$.

Proof: We have from (2.8):

$$
\begin{aligned}
& Z(x,-\xi) \hat{\phi}(\xi) \\
& =\left|m_{0}(\xi / 2)\right|^{2} \bar{\phi}(\xi / 2) Z(2 x,-\xi / 2)+\overline{m_{0}}(\xi / 2) \overline{\hat{\phi}}(\xi / 2) \sum_{\epsilon \neq 0} m_{0}(\pi \epsilon+\xi / 2) Z(2 x,-\xi / 2-\pi \epsilon),
\end{aligned}
$$

and so

$$
\begin{aligned}
& Z(x,-\xi) \hat{\hat{\phi}}(\xi)-Z(2 x,-\xi / 2) \overline{\hat{\phi}}(\xi / 2) \\
& =\left(\left|m_{0}(\xi / 2)\right|^{2}-1\right) \overline{\hat{\phi}}(\xi / 2) Z(2 x,-\xi / 2)+\overline{m_{0}}(\xi / 2) \overline{\hat{\phi}}(\xi / 2) \sum_{\epsilon \neq 0} m_{0}(\pi \epsilon+\xi / 2) Z(2 x,-\xi / 2-\pi \epsilon) .
\end{aligned}
$$

We now use (6.6) and $(i)$ and $(i i)$ of Lemma 6.1 (with $g$ and $h$ as in the Lemma), noting $\|\tilde{E}(2 x, \xi / 2)\|_{\infty}=\|\tilde{E}(x, \xi / 2)\|_{\infty}$, to obtain 


$$
\begin{aligned}
\left(\int_{|\xi|<1} \mid\right. & \left.\|\tilde{E}(x, \xi)\|_{\infty}-\|\tilde{E}(2 x, \xi / 2)\|_{\infty} \mid h(\xi) d \xi\right)^{1 / 2} \\
=\left(\int_{|\xi|<1} \mid\right. & \left.\left\|e^{-i x \xi} Z(x,-\xi) \hat{\phi}(\xi)-(2 \pi)^{-d / 2}\right\|_{\infty}-\left\|e^{-i x \xi} Z(2 x,-\xi / 2) \overline{\hat{\phi}}(\xi / 2)-(2 \pi)^{-d / 2}\right\|_{\infty} \mid{ }^{2} h(\xi) d \xi\right)^{1 / 2} \\
\leq & \left(\int_{|\xi|<1}\|Z(x,-\xi) \hat{\phi}(\xi)-Z(2 x,-\xi / 2) \hat{\phi}(\xi / 2)\|_{\infty}^{2} h(\xi) \mathrm{d} \xi\right)^{1 / 2} \\
= & \left(\int_{|\xi|<1} \|\left(\left|m_{0}(\xi / 2)\right|^{2}-1\right) \overline{\hat{\phi}}(\xi / 2) Z(2 x,-\xi / 2)\right. \\
& \left.+\overline{m_{0}}(\xi / 2) \hat{\hat{\phi}}(\xi / 2) \sum_{\epsilon \neq 0} m_{0}(\pi \epsilon+\xi / 2) Z(2 x,-\xi / 2-\pi \epsilon) \|{ }_{\infty}^{2} h(\xi) d \xi\right)^{1 / 2} \\
\leq & \left(\int_{|\xi|<1}\left|\left(\left|m_{0}(\xi / 2)\right|^{2}-1\right) \overline{\hat{\phi}}(\xi / 2)\|Z(2 x,-\xi / 2)\|_{\infty}\right|^{2} h(\xi) d \xi\right)^{1 / 2} \\
& +\left(\int_{|\xi|<1}\left|\overline{\mathrm{m}_{0}}(\xi / 2) \overline{\hat{\phi}}(\xi / 2) \sum_{\epsilon \neq 0}\right| m_{0}(\pi \epsilon+\xi / 2)\left|\|Z(2 x,-\xi / 2-\pi \epsilon)\|_{\infty}\right|^{2} h(\xi) d \xi\right)^{1 / 2} \\
< & \infty,
\end{aligned}
$$

where we have used that $Z(x, \xi), \widehat{\phi}(\xi)$, and $m_{0}(\xi)$ are uniformly bounded in $x$ and $\xi$.

Lemma 6.3: Assume that $\sigma>d / 2$. There exists a number $C>1$ such that for any $g_{1}(|\xi|) \in \mathcal{L}_{C}\left(\mathbb{R}^{d}\right)$, the following holds (defining $\left.h_{1}(\xi)=g_{1}(\xi)|\xi|^{-2 \sigma}\right)$ :

For any positive $Y(\xi)$ with $Y(\xi) \underset{\xi \rightarrow 0}{\longrightarrow} 0$, if $\int_{|\xi|<1}|Y(\xi)-Y(\xi / 2)|^{2} h_{1}(\xi) d \xi<\infty$, then $\int_{|\xi|<1}\left|Y^{2}(\xi)\right| h_{1}(\xi) d \xi<\infty$

Proof: Define $g(|\xi|)=\left\{\begin{array}{ll}g_{1}(|\xi|) & \text { if }|\xi|<1 \\ 0 & \text { otherwise }\end{array}\right.$, with $g_{1} \in \mathcal{L}_{C}$, and $C$ to be determined. Let $h(\xi)=g(|\xi|)|\xi|^{-2 \sigma}$, so that from the assumption of the Lemma

$$
\int_{\mathbb{R}^{\mathrm{d}}}|Y(\xi)-Y(\xi / 2)|^{2} h(\xi) \mathrm{d} \xi<\infty .
$$

Now choose $C>1$ so

$$
2^{-\sigma} C^{1 / 2}<2^{-d / 2}
$$

which is possible since $\sigma>d / 2$.

Then

$$
\int_{\mathbb{R}^{d}}|Y(\xi)-Y(\xi / 2)|^{2} h(\xi) d \xi=\int_{\mathbb{R}^{d}}\left|Y(\xi) h(\xi)^{1 / 2}-Y(\xi / 2) h(\xi / 2)^{1 / 2} r(\xi / 2)\right|^{2} d \xi
$$

where (recall $g \in \mathcal{L}_{C}$; see Definitions 3.3) 


$$
r(\xi / 2) \equiv h(\xi)^{1 / 2} / h(\xi / 2)^{1 / 2}=2^{-\sigma} \frac{g(|\xi|)^{1 / 2}}{g(|\xi / 2|)^{1 / 2}} \leq 2^{-\sigma} C^{1 / 2}<2^{-d / 2} .
$$

We have defined $0 / 0=0$ above.

Let $Y_{n}(\xi)$ be a positive sequence in $L^{2}$ which converges pointwise to $Y(\xi)$ such that $Y_{n}(\xi) \leq Y(\xi)$. We may assume convergence occurs such that, defining $G(\xi)=Y(\xi) h(\xi)^{1 / 2}$ and $G_{n}(\xi)=Y_{n}(\xi) h(\xi)^{1 / 2}$,

$$
\left|G_{n}(\xi)-r(\xi / 2) G_{n}(\xi / 2)\right| \leq|G(\xi)-r(\xi / 2) G(\xi / 2)| .
$$

For example, since $Y(\xi) \underset{\xi \rightarrow 0}{\longrightarrow} 0$, we could choose $Y_{n}(\xi)=(Y(\xi)-1 / n)_{+}$, with + denoting the greater of the argument and 0 . Then $\left|Y_{n}(\alpha)-Y_{n}(\beta)\right| \leq|Y(\alpha)-Y(\beta)|$ for all $\alpha, \beta \in \mathbb{R}^{d}$, and

$$
\begin{aligned}
\left|G_{n}(\xi)-r(\xi / 2) G_{n}(\xi / 2)\right| & =\left|Y_{n}(\xi) h(\xi)^{1 / 2}-Y_{n}(\xi / 2) h(\xi)^{1 / 2}\right| \\
& \leq\left|Y(\xi) h(\xi)^{1 / 2}-Y(\xi / 2) h(\xi)^{1 / 2}\right| \\
& =|G(\xi)-r(\xi / 2) G(\xi / 2)|
\end{aligned}
$$

as desired. Now

$$
\begin{aligned}
\left\|G_{n}(\xi)-r(\xi / 2) G_{n}(\xi / 2)\right\|_{2} & \geq\left\|G_{n}(\xi)\right\|_{2}-\left\|r(\xi / 2) G_{n}(\xi / 2)\right\|_{2} \\
& \geq\left(1-2^{d / 2-\sigma} C^{1 / 2}\right)\left\|G_{n}(\xi)\right\|_{2}
\end{aligned}
$$

By our choice of $C$ we have $1-2^{d / 2-\sigma} C^{1 / 2}>0$. By (6.7)

$$
\int_{\mathbb{R}^{d}}|G(\xi)-r(\xi / 2) G(\xi / 2)|^{2} d \xi=\int_{\mathbb{R}^{\mathrm{d}}}|Y(\xi)-Y(\xi / 2)|^{2} h(\xi) \mathrm{d} \xi<\infty .
$$

Thus by dominated convergence and (6.8), the left side of (6.9) converges, so the right side is bounded in $n$. Thus the sequence $\left\|G_{n}(\xi)\right\|_{2}$ is bounded, and since $G_{n}(\xi)$ converges to $G(\xi)$ pointwise from below, $\|G(\xi)\|_{2}$ is finite, proving the lemma.

Recall for an inner product space $V$ (with inner product $\cdot$ ), a family of vectors $\left\{Z^{i}\right\} \subset V$ forms a frame if there exist constants $A>0$ and $B<\infty$ such that for all $f \in V$,

$$
A\|f\|^{2} \leq \sum_{i}\left|f \cdot Z^{i}\right|^{2} \leq B\|f\|^{2} .
$$

Lemma 6.4: In a finite dimensional space, the optimal frame bound is a continuous function of the frame. Specifically, if the vectors $\left\{Z^{i}(\xi)\right\}_{i=1}^{m}$ form a frame in $\mathbb{R}^{n}$ for each $\xi$, and if the $\boldsymbol{Z}^{i}(\xi)$ vary continuously in $\xi$, then

$$
\sup _{\boldsymbol{f} \in \mathbb{R}^{n}} \frac{\sum_{i=}^{m}\left|\boldsymbol{f} \cdot \boldsymbol{Z}^{i}(\xi)\right|^{2}}{|\boldsymbol{f}|^{2}}, \quad \inf _{\boldsymbol{f} \in \mathbb{R}^{n}} \frac{\sum_{i=1}^{m}\left|\boldsymbol{f} \cdot \boldsymbol{Z}^{i}(\xi)\right|^{2}}{|\boldsymbol{f}|^{2}}
$$

are continuous functions of $\xi$. 
Proof: We write

$$
\sup _{\boldsymbol{f} \in \mathbb{R}^{n}} \frac{\sum_{i=1}^{m}\left|\boldsymbol{f} \cdot \boldsymbol{Z}^{i}(\xi)\right|^{2}}{|\boldsymbol{f}|^{2}}=\sup _{\boldsymbol{f} \in \mathbb{R}^{n},|f|=\mathbf{1}} \sum_{i=1}^{m}\left|\boldsymbol{f} \cdot \boldsymbol{Z}^{i}(\xi)\right|^{2} .
$$

Because suprema of equicontinuous function families are continuous, it is only necessary that we check that the family $\left\{\sum_{i=1}^{m}\left|\boldsymbol{f} \cdot \boldsymbol{Z}^{i}(\xi)\right|^{2}\right\}_{\boldsymbol{f} \in S}$ is equicontinuous, where $S=\{f:|f|=1\}$. But for this it suffices to show that for each $i,\left\{\left|\boldsymbol{f} \cdot \boldsymbol{Z}^{i}(\xi)\right|^{2}\right\}_{\boldsymbol{f} \in S}$ forms an equicontinuous family of functions, which is clear by the Schwartz inequality.

Lemma 6.5: Let $\boldsymbol{f}(\xi)$ and $\boldsymbol{Z}(x, \xi)$ be vector functions on $\mathbb{R}^{d}$, let $\alpha>0$, and assume

(i) The vectors $\{\boldsymbol{Z}(x, 0)\}$ span $\mathbb{R}^{n}$, i.e., there is no nonvanishing vector $\boldsymbol{d}$ such that $\boldsymbol{Z}(x, 0) \cdot \boldsymbol{d}=0$ a.e. $[x]$.

(ii) $\boldsymbol{Z}(x, \xi)$ is continuous in $\xi$.

Then if $\boldsymbol{f}(\xi)$ is a vector function in $L^{2}\left(\mathbb{R}^{n}\right)$ such that

$$
\int_{|\xi|>c}|\boldsymbol{f}(\xi) \cdot \boldsymbol{Z}(x, \xi)|^{2} d \xi=O\left(c^{-\alpha}\right) \quad \text { a.e. }[x],
$$

it follows that

$$
\int_{|\xi|>c}|\boldsymbol{f}(\xi)|^{2} d \xi=O\left(c^{-\alpha}\right) .
$$

Proof: Assume (6.12). Let $\left\{x_{i}\right\}_{i=1}^{n}$ be such that $\left\{Z\left(x_{i}, 0\right)\right\}_{i}$ is a basis for $\mathbb{R}^{n}$, and such that the equality in (6.12) holds for $x=x_{i} \forall i$. Then by the previous Lemma, for $|\xi|$ sufficiently small, say $|\xi|<\delta$,

$$
|f(\xi)|^{2} \leq K \sum_{i}\left|\boldsymbol{f}(\xi) \cdot \boldsymbol{Z}\left(x_{i}, \xi\right)\right|^{2}
$$

since $\left\{\boldsymbol{Z}\left(x_{i}, 0\right)\right\}_{i}$ forms a basis and thus a frame.

Now write (for small $c$ )

$$
\begin{aligned}
\int_{|\xi|>c}|\boldsymbol{f}(\xi)|^{2} d \xi & =\int_{\delta>|\xi|>c}|\boldsymbol{f}(\xi)|^{2} d \xi+\int_{|\xi|>\delta}|\boldsymbol{f}(\xi)|^{2} d \xi \\
& \leq \int_{\delta>|\xi|>c} K \sum_{i}\left|\boldsymbol{f}(\xi) \cdot \boldsymbol{Z}\left(x_{i}, \xi\right)\right|^{2} d \xi+\int_{|\xi|>\delta}|\boldsymbol{f}(\xi)|^{2} d \xi \\
& \leq O\left(c^{-\alpha}\right)+K_{1} \\
& =O\left(c^{-\alpha}\right) .
\end{aligned}
$$

\section{Proof of Theorem 5}

The next theorem establishes the equivalence of the functions $I_{s}$ and $L_{s}$. 
Theorem 7.1: For $\alpha>0,0<c<1, t \neq d+\alpha$,

$$
\int_{c<|\xi|<1}\left(1-(2 \pi)^{d / 2}|\hat{\phi}(\xi)|\right)|\xi|^{-t} d \xi=O\left(c^{-\alpha}\right)
$$

if and only if

$$
\int_{c<|\xi|<1}\left(1-\left|m_{0}(\xi)\right|^{2}\right)|\xi|^{-\mathrm{t}} d \xi=O\left(c^{-\alpha}\right)
$$

Proof: Let the left side of (7.1) be $T(c)$ and that of (7.2) be $U(c)$. Recall $\widehat{\phi}(\xi)=m_{0}(\xi / 2) \widehat{\phi}(\xi / 2)$. Let $S(c) \equiv \int_{c<|\xi|<1}\left(1-(2 \pi)^{d}|\widehat{\phi}(\xi)|^{2}\right)|\xi|^{-t} d \xi$. Note by factoring the integrand that $S(c)=O\left(c^{-\alpha}\right)$ iff $T(c)=O\left(c^{-\alpha}\right)$.

Assume (7.1). Then (6.2) gives (7.2) (after factoring the differences of squares on the right of (6.2)).

Conversely assume (7.2) holds, i.e., that $U(c)=O\left(c^{-\alpha}\right)$. Then note

$$
\left(1-(2 \pi)^{d}|\widehat{\phi}(2 \xi)|^{2}\right)-\left(1-(2 \pi)^{d}|\widehat{\phi}(\xi)|^{2}\right)=(2 \pi)^{d}\left(1-\left|m_{0}(\xi)\right|^{2}\right)|\widehat{\phi}(\xi)|^{2} .
$$

The factors in the integrand of (7.2) are positive since they symbol $m_{0}(\xi)$ assumes its maximum of 1 at $\xi=0$. Thus, without loss redefining $1-(2 \pi)^{d / 2}|\widehat{\phi}(\xi)|^{2}=0$ for $|\xi|>1 / 2$

$$
\begin{aligned}
& O\left(c^{-\alpha}\right)=\int_{c<|\xi|<1}\left|\left(1-(2 \pi)^{d}|\hat{\phi}(\xi)|^{2}\right)-\left(1-(2 \pi)^{d}|\hat{\phi}(\xi / 2)|^{2}\right)\right||\xi|^{-t} d \xi \\
& \quad \geq\left.\left|\int_{c<|\xi|<1}\left(1-(2 \pi)^{d}|\hat{\phi}(\xi)|^{2}\right)\right| \xi\right|^{-t} d \xi-2^{d-t} \int_{c / 2<|\xi|<1 / 2}\left(1-(2 \pi)^{d}|\hat{\phi}(\xi)|^{2}\right)|\xi|^{-t} d \xi \mid \\
& \quad=\left|S(c)-2^{d-t} S(c / 2)\right| .
\end{aligned}
$$

Defining $P(c)=c^{t-d} S(c)$ we have

$$
O\left(c^{-\alpha}\right)=\left|c^{d-t}(P(c)-P(c / 2))\right|,
$$

so

$$
|P(c)-P(c / 2)|=O\left(c^{-\alpha+t-d}\right) .
$$

Hence if $t-\alpha-d<0$, by Lemma 3.2, $P(c)=O\left(c^{t-\alpha-d}\right)$, so $T(c)=O\left(c^{-\alpha}\right)$, as desired.

On the other hand, if $t-\alpha-d>0$, then we can again apply Lemma 3.2 (a) if we can show $P(0) \equiv \lim _{c \rightarrow 0} P(c)=0$. To this end, note that since $\left(1-(2 \pi)^{d / 2}|\hat{\phi}(\xi)|\right) \underset{\xi \rightarrow 0}{\longrightarrow} 0$,

$$
\int_{c / 2<|\xi|<c}\left(1-(2 \pi)^{d / 2}|\hat{\phi}(\xi)|\right)|\xi|^{-t} d \xi \leq(c / 2)^{-t}|B(c)| \sup _{|\xi|<c}\left(1-(2 \pi)^{d / 2}|\widehat{\phi}(\xi)|\right)=o\left(c^{d-t}\right),
$$


where $|B(c)|$ denotes the volume in $d$ dimensions of the ball of radius $c$. Thus by the second paragraph of the statement of Theorem 3.4 (note since $t-\alpha-d>0$ we have $t>d$ ) relating to replacement of $O$ by $o$,

$$
T(c)=\int_{c<|\xi|<1}\left(1-(2 \pi)^{d / 2}|\hat{\phi}(\xi)|\right)|\xi|^{-t} d \xi=o\left(c^{d-t}\right) .
$$

So by factoring the integrand below,

$$
S(c)=\int_{c<|\xi|<1}\left(1-(2 \pi)^{d}|\hat{\phi}(\xi)|^{2}\right)|\xi|^{-t} d \xi=o\left(c^{d-t}\right),
$$

and thus $P(c)=c^{t-d} S(c) \underset{c \rightarrow 0}{\longrightarrow} 0$. Thus we can apply Lemma 3.2 to (7.4), to again obtain $P(c)=O\left(c^{t-\alpha-d}\right)$, and $T(c)=O\left(c^{-\alpha}\right)$, completing the proof.

Note for later reference that

$$
\mathcal{F}\left(\psi_{j k}^{\lambda}(x)\right)=2^{-j d / 2} e^{-i 2^{-j} \xi k} \hat{\psi}^{\lambda}\left(2^{-j} \xi\right)
$$

Recall also formula (2.6) for $\widetilde{E}(x, \xi)$.

We now give the complete proof of Theorem 5, the main result of this paper.

\section{Proof of Theorem 5:}

(o) If $\sigma=0$ it suffices to show that we fail to have any positive order of convergence in $H^{s}$ for $s>d / 2$. Recall $\sigma=0$ means $I-P: H_{h}^{s} \rightarrow L^{\infty}$ is unbounded for all $s \geq 0$.

To begin we claim that in this case the convergence rate of the MRA for $s>d / 2$ is independent of $s$. The rest of the proof is similar to that of Theorem 4.3.

Assume we have approximation order $r>0$ in $H^{s}$. Then uniformly (as usual a.e.) in $x$, we have by Theorem 4.2

$$
\int|\widetilde{E}(x, \xi)|^{2}(c+|\xi|)^{-2 s} \mathrm{~d} \xi \leq K c^{-2 s+2 r+d}=K c^{-\eta} .
$$

Assume for the moment that $s>d / 2+1 / 2$. We apply Corollary 3.6 with $f_{x}^{*}(\xi)=|\widetilde{E}(x, \xi)|^{2}, \quad \gamma_{1}=-2 s, \eta=2 s-2 r-d$, and $\delta=1+2 \epsilon$, with $\epsilon>0$ to be determined below. Note that the supremum of the left side of (7.5) diverges as $c \rightarrow 0$ by Corollary 2.4, the equivalence of $\left(a^{\prime}\right)$ and $(b)$ in Theorem 1 and the fact that $s>d / 2>\sigma$, so that $-\eta<0$. Similarly to the proof of Theorem 4.3, it follows from Corollary 3.6 that for some $\epsilon>0$ we have uniformly a.e. in $x$ :

$$
\left.\int \widetilde{E}(x, \xi)\right|^{2}(c+|\xi|)^{-2(s-(1 / 2+\epsilon))} d \xi \leq K c^{-2(s-(1 / 2+\epsilon))+2 r+d}=K c^{1-\eta+2 \epsilon} .
$$

More precisely, we choose $\epsilon$ so small that $s-(1 / 2+\epsilon)>d / 2$, and also so that the exponent $1-\eta+2 \epsilon \neq 0$. Then as in the previous proof, we conclude that in fact 
$1-\eta+2 \epsilon<0$, since the left side of (7.6) diverges as $c \rightarrow 0$. The divergence of the left side of (7.6) follows by Corollary 2.4, the equivalence of $\left(a^{\prime}\right)$ and $(b)$ in Theorem 1, and the fact that $s-(1 / 2+\epsilon)>d / 2>\sigma$. Corollary 3.6 then gives (7.6).

From this we conclude we have pointwise approximation order $r$ in $H^{s-(1 / 2+\epsilon)}$. With appropriate choice of $\epsilon>0$, it follows (as in the proof of Theorem 4.3) that the best pointwise approximation order $r$ (as a function of $s$ ) is periodic with period $1 / 2$ and nondecreasing. Thus the set of orders is constant for $s>d / 2$, as desired.

We have established the rate of convergence $r$ in $H^{s}$ is independent of $s$. By Theorem 1 , it is also less than $s-d / 2$ in $H^{s}$ for $s>d / 2$. Since $s$ can be arbitrarily close to $d / 2$, we conclude $r<s-d / 2$ must be smaller than any positive number. Hence there is no positive rate of convergence $r$ in any Sobolev space $H^{s}$ with $s>d / 2$. Since for $s^{\prime} \leq d / 2$ and $s>d / 2$ we have $H^{s^{\prime}} \supset H^{s}$, it also follows that there are no positive convergence rates for $s^{\prime} \leq d / 2$. Thus we have proved $(o)$.

(i) For $0 \leq s \leq d / 2$, there exist unbounded $f \in H^{s}$. However, for any $f \in H^{s}$, Pf $\in L^{\infty}$. This follows since by our assumptions, the scaling function satisfies $|\phi(x)| \leq \eta(|x|)$ with $\eta$ bounded, decreasing, and in $L^{1}\left(\mathbb{R}^{d}\right)$. Thus

$$
|P f|=\left|\sum_{k} a_{k} \phi(x-k)\right| \leq\left(\sum_{k}\left|a_{k}\right|^{2}\right)^{1 / 2}\left(\sum_{k} \eta(x-k)^{2}\right)^{1 / 2} \leq K\|P f\|_{L^{2}}\|\eta\|_{2} .
$$

Above, the sum involving $\eta$ can be bounded by the norm on the right because it can be bounded by an integral. Thus for unbounded $f \in H^{s}$ we have $E f=f-P f$ is unbounded. Therefore $E: H^{s} \rightarrow L^{\infty}$ is never bounded, and hence $\left\|E_{n}\right\|_{H^{s} \rightarrow L^{\infty}}$ fails to have any decay rate. This yields approximation order 0 in these spaces (by our definition of approximation order 0$)$.

(ii) This follows from equivalence of $\left(a^{\prime \prime}\right)$ and $(b)$ in Theorem 1.

(iii) This follows from the equivalence of $(a)$ and $\left(d^{\prime}\right)$ of Theorems 1 and 3. Specifically if $I_{\sigma}(0)<\infty$ then by these theorems the best pointwise approximation order in $H^{\sigma}$ is $\sigma-d / 2$. But if $I_{\sigma}(0)=\infty$, then Theorems 1 and 3 imply the best approximation order in $H^{\sigma}$ cannot be $\sigma-d / 2$. However for $s=\sigma-\epsilon$ Theorem 1 implies the best approximation order in $H^{s}$ is $s-d / 2=\sigma-d / 2-\epsilon$. Thus for any $s>\sigma-\epsilon$ the best approximation order is at least $\sigma-d / 2-\epsilon$. Since this holds for all $\epsilon>0$, for $s>\sigma$ the MRA has approximation order $\sigma-d / 2-\epsilon$ for all $\epsilon>0$, and hence has best order $(\sigma-d / 2)^{-}$as desired.

(iv) Since $\sigma \neq 0$, we have $\sigma>d / 2$. By Theorem 4.3 the best approximation order in $H^{s}$ is independent of $s$ for $s>\sigma$. Thus to determine this order for any $s>\sigma$ we only consider $s=\sigma+1 / 2$. By part $($ iii $)$ of this Theorem, since best approximation order in $H^{r_{2}}$ cannot be worse than in $H^{r_{1}}$ for $r_{2}>r_{1}$, the best order in $H^{s}$ is at least $(\sigma-\mathrm{d} / 2)^{-}$.

We now show that if 


$$
I_{\sigma+1 / 2}(c) \equiv \int_{1 \geq|\xi| \geq c}\left(1-(2 \pi)^{d / 2}|\hat{\phi}(\xi)|\right)|\xi|^{-2 \sigma-1} d \xi=O(1 / c)
$$

then we have approximation order $\sigma-d / 2$ in $H^{\mathrm{s}}$ for $s>\sigma$. To do this we will verify

$$
J_{\sigma+1 / 2}(c) \equiv \sup _{x} \int_{|\xi| \geq c}|\tilde{E}(x, \xi)|^{2}|\xi|^{-2 \sigma-1} d \xi=O(1 / c),
$$

and use Theorem 5.1.

To verify (7.8) we will use equivalence of $(a)$ and $\left(c^{\prime}\right)$ in Corollary 3.5. Specifically we let $f_{x}(\xi)=|\tilde{E}(x, \xi)|^{2}|\xi|^{-2 \sigma-1}$ and $\alpha=1$. It then suffices to show for some fixed $C>1$ that for any $g_{1}(|\xi|) \in \mathcal{L}_{C}$ such that $\int|\xi|^{-d} g_{1}(|\xi|) d \xi<\infty$,

$$
\int_{|\xi| \leq 1} g_{1}(|\xi|) \sup _{x}|\tilde{E}(x, \xi)|^{2}|\xi|^{-2 \sigma} d \xi<K<\infty
$$

To prove (7.9) for some $C$ (we will choose $C$ later), let $g(|\xi|)=\left\{\begin{array}{ll}g_{1}(|\xi|) & \text { if }|\xi|<1 \\ 0 & \text { otherwise }\end{array}\right.$. Defining

$$
Y(\xi) \equiv\|\tilde{E}(x, \xi)\|_{\infty}
$$

we have by Lemma 6.2 that if $h(\xi) \equiv g(|\xi|)|\xi|^{-2 \sigma}$,

$$
\int_{|\xi|<1}|Y(\xi)-Y(\xi / 2)|^{2} h(\xi) d \xi<\infty .
$$

Note that $Y(\xi) \underset{\xi \rightarrow 0}{\longrightarrow} 0$ (see remarks before equation (2.3.16) in [KR1]). Now choose $C$ as in the statement of Lemma 6.3. Then, using Lemma 6.3, we have

$$
\int_{|\xi|<1} g(|\xi|) \sup _{x}|\tilde{E}(x, \xi)|^{2}|\xi|^{-2 \sigma} d \xi=\int_{|\xi|<1} Y^{2}(\xi) h(\xi) d \xi<\infty
$$

so (7.9) has been established, proving we have approximation order $\sigma-d / 2$ in $H^{s}$ for $s>\sigma$.

To prove the second case of $(i v)$, suppose now that

$$
I_{\sigma+1 / 2}(c) \equiv \int_{1 \geq|\xi| \geq c}\left(1-(2 \pi)^{d / 2}|\hat{\phi}(\xi)|\right)|\xi|^{-2 \sigma-1} \mathrm{~d} \xi \neq O(1 / c)
$$

for $c<1$. By the equivalence of $(a)$ and $(c)$ in Theorem 3.4 for any $C>1$ there then exists $g(|\xi|) \in \mathcal{L}_{C}$ with $\int|\xi|^{-d} g(|\xi|) d \xi<\infty$ and

$$
\int_{|\xi|<1}\left(1-(2 \pi)^{d / 2}|\hat{\phi}(\xi)|\right)|\xi|^{-2 \sigma} g(|\xi|) d \xi=\infty .
$$


We wish to show that in this case the order of convergence in $H^{s}$ for $s>\sigma$ is $(\sigma-d / 2)^{-}$. To this end it suffices to show, by Theorem 5.1, that $J_{\sigma+1 / 2}(c) \neq O(1 / c)$ for $c \leq 1$. Again let $h(\xi)=g(|\xi|)|\xi|^{-2 \sigma}$.

We have

$$
\int_{|\xi|<1}\left(1-(2 \pi)^{d / 2}|\hat{\phi}(\xi)|\right) h(\xi) d \xi=\infty
$$

Letting $C$ denote the unit cube in $\mathbb{R}^{d}$, we have by (2.9) and (2.10):

$$
\begin{aligned}
& \int_{C} d x \int_{|\xi|<1}\left|\tilde{E}_{0}(x, \xi)\right|^{2} h(\xi) d \xi \\
= & \int_{C} d x \int_{|\xi|<1}\left|(2 \pi)^{-d / 2}\left(1-(2 \pi)^{d}|\hat{\hat{\phi}}(\xi)|^{2}\right)-(2 \pi)^{d / 2} \sum_{l \neq 0} \hat{\hat{\phi}}(\xi) \hat{\phi}(2 \pi l+\xi) e^{2 \pi \mathrm{ix} l}\right|^{2} h(\xi) d \xi \\
= & \int_{|\xi|<1}\left(\left|(2 \pi)^{-d / 2}\left(1-(2 \pi)^{d}|\hat{\phi}(\xi)|^{2}\right)\right|^{2}+\sum_{l \neq 0}\left|(2 \pi)^{d / 2} \hat{\hat{\phi}}(\xi) \hat{\phi}(2 \pi l+\xi)\right|^{2}\right) h(\xi) d \xi \\
\geq & \int_{|\xi|<1} \sum_{l \neq 0}\left|(2 \pi)^{d / 2} \hat{\hat{\phi}}(\xi) \hat{\phi}(2 \pi l+\xi)\right|^{2} h(\xi) d \xi
\end{aligned}
$$

The second equality follows from the Parseval identity for Fourier series, since the $x$ integration (once $x$ and $\xi$ integrations are interchanged) is the square of the $L^{2}$-norm of a Fourier series in $x$.

By (6.4) and (7.10), factoring the difference of squares below,

$$
(2 \pi)^{d} \int_{|\xi|<1}\left(\sum_{\ell \neq 0}|\widehat{\phi}(\xi+2 \pi \ell)|^{2}\right) h(\xi) d \xi=\int_{|\xi|<1}\left(1-(2 \pi)^{d}|\hat{\phi}(\xi)|^{2}\right) h(\xi) d \xi=\infty
$$

so comparing with $(7.11)$, (since $\widehat{\phi}(0) \neq 0$ and $\widehat{\phi}$ is continuous)

$$
\int_{C} d x \int_{|\xi|<1}\left|\tilde{E}_{0}(x, \xi)\right|^{2} h(\xi) d \xi=\infty
$$

The above is an $L^{1}$ norm over the unit cube $C$ in $x$, so the $L^{\infty}$ norm is also infinite.

Thus the error operator $E$ with Fourier kernel

$$
\tilde{E}_{0}(x, \xi)=(2 \pi)^{-d / 2} e^{i x \xi}-Z(x,-\xi) \overline{\hat{\phi}}(\xi)
$$

satisfies

$$
\left\|\int_{|\xi|<1}\left|\tilde{E}_{0}(x, \xi)\right|^{2} h(\xi) d \xi\right\|_{\infty}=\infty
$$


But by the equivalence of parts $(a)$ and $\left(c^{\prime}\right)$ of Corollary 3.5, letting $f_{x}(\xi)=\left|\tilde{E}_{0}(x, \xi)\right|^{2}|\xi|^{-2 \sigma-1}$, it follows

$$
J_{\sigma+1 / 2} \equiv\left\|\int_{c \leq|\xi|}\left|\tilde{E}_{0}(x, \xi)\right|^{2}|\xi|^{-2 \sigma-1} d \xi\right\|_{\infty} \neq O(1 / c) .
$$

Now by Theorem 5.1, it follows that the best pointwise order of approximation in $H^{s}$ is $(\sigma-d / 2)^{-}$, as desired. This completes the proof of $(i v)$.

(v) We wish to show the above statements hold with $I_{\sigma+1 / 2}(c)$ replaced by $K_{\sigma+1 / 2}(c)$ or $L_{\sigma+1 / 2}(c)$. Note by Theorems 1 through 4 the proof of $(i i i)$ does not change if we replace $I$ by $K$ or $L$.

Now consider the proof of $(i v)$ in these cases, first with replacement of $I_{\sigma+1 / 2}(c)$ with $L_{\sigma+1 / 2}(c)$. We wish to show

$$
I_{\sigma+1 / 2}(c) \equiv \int_{1 \geq|\xi| \geq c}\left(1-(2 \pi)^{\mathrm{d} / 2}|\hat{\phi}(\xi)|\right)|\xi|^{-2 \sigma-1} d \xi=O(1 / c),
$$

if and only if

$$
L_{\sigma+1 / 2}(c) \equiv \int_{1 \geq|\xi| \geq c}\left(1-\left|m_{0}(\xi)\right|^{2}\right)|\xi|^{-2 \sigma-1} d \xi=O(1 / c) .
$$

We apply Theorem 7.1 with $t=2 \sigma+1$, and $\alpha=1$, so $d+\alpha=d+1$. We have $2 \sigma>d$, so that $t=2 \sigma+1 \neq d+\alpha$. It follows that $I_{\sigma+1 / 2}(c)$ may be replaced by $L_{\sigma+1 / 2}(c)$, as desired.

To show we can replace $I_{\sigma+1 / 2}(c)$ by $K_{\sigma+1 / 2}(c)$, we show first that if

$$
I_{\sigma+1 / 2}(c)=O(1 / c)
$$

then the same holds for

$$
K_{\sigma+1 / 2}(c) \equiv \sup _{\lambda} \int_{1 \geq|\xi| \geq c}\left|\psi^{\lambda}(\xi)\right|^{2}|\xi|^{-2 \sigma-1} d \xi .
$$

Note that if (7.13) holds then by part $(i v)$ of this theorem and by Theorem 5.1,

$$
J_{\sigma+1 / 2} \equiv\left\|\int_{|\xi|>c}\left|\widetilde{E}_{0}(x, \xi)\right|^{2}|\xi|^{-2 \sigma-1} d \xi\right\|_{\infty}=O(1 / c)
$$

which by Proposition 4.1 implies that

$$
\left\|\int_{|\xi|>c}\left|\widehat{Q}_{0}(x, \xi)\right|^{2}|\xi|^{-2 \sigma-1} d \xi\right\|_{\infty}=O(1 / c) .
$$

We write $Q=Q_{0}$ and $E=E_{0}$. Note that $\mathcal{F}\left(\bar{\psi}^{\lambda}(y-k)\right)=\overline{\widehat{\psi}}^{\lambda}(-\xi) e^{-i \xi k}$. We then have that the kernel [KKR1] 


$$
\begin{aligned}
\widehat{Q}(x, \xi) & =\mathcal{F}_{y}(Q(x, y)) \\
& =\mathcal{F}_{y}\left(\sum_{k, \lambda} \psi^{\lambda}(x-k) \bar{\psi}^{\lambda}(y-k)\right) \\
& =\sum_{k, \lambda} \psi^{\lambda}(x-k) \mathcal{F}_{y}\left(\bar{\psi}^{\lambda}(y-k)\right) \\
& =\sum_{k, \lambda} \psi^{\lambda}(x-k) \overline{\widehat{\psi}}^{\lambda}(-\xi) e^{-i \xi k} \\
& =\sum_{\lambda} \overline{\widehat{\psi}}^{\lambda}(-\xi) Z^{\lambda}(x, \xi),
\end{aligned}
$$

where $Z^{\lambda}(x, \xi)=\sum_{k} \psi^{\lambda}(x+k) e^{i \xi k}$ is the Zak transform of $\psi^{\lambda}$. But for a set $G$ of $x$ of positive measure, $Z^{\lambda}(x, \xi)$ is nonzero at $\xi=0$ (see [KR1] after (2.2.9)), and it is always continuous in $\xi$, since its Fourier coefficients $\psi^{\lambda}(x+k)$ are in $\ell^{1}$. We have, letting $\boldsymbol{Z}(x, \xi)=\left(Z^{1}, Z^{2}, \ldots, Z^{\ell}\right)$ and $\boldsymbol{\psi}(\xi) \equiv|\xi|^{-\sigma-1 / 2}\left(\overline{\widehat{\psi}}^{1}(-\xi), \overline{\widehat{\psi}}^{2}(-\xi), \ldots, \overline{\widehat{\psi}}^{\ell}(-\xi)\right)$,

$$
\begin{aligned}
\int_{|\xi|>c}|\boldsymbol{\psi}(\xi) \cdot \boldsymbol{Z}(x, \xi)|^{2} d \xi & =\int_{|\xi|>c}\left|\sum_{\lambda}{\overline{\widehat{\psi}^{\lambda}}}^{\lambda}(-\xi) Z^{\lambda}(x, \xi)\right|^{2}|\xi|^{-2 \sigma-1} d \xi \\
& =\int_{|\xi|>c}\left|\widehat{Q}_{0}(x, \xi)\right|^{2}|\xi|^{-2 \sigma-1} d \xi \\
& =O(1 / c) .
\end{aligned}
$$

The set $\{\boldsymbol{Z}(x, 0)\}_{x \in G}$ spans $\mathbb{R}^{d}$ in that there is no nonvanishing vector $\boldsymbol{d}$ (see [KR1], before (2.2.9)) such that $\boldsymbol{Z}(x, 0) \cdot \boldsymbol{d}=0$ for almost all $x \in G$. Thus we may apply Lemma 6.5 to conclude that

$$
\int_{|\xi|>c}|\psi(\xi)|^{2} d \xi=O(1 / c)
$$

Thus

$$
K_{\sigma+1 / 2}(c)=\sup _{\lambda} \int_{1>|\xi|>c}\left|\psi^{\lambda}(\xi)\right|^{2}|\xi|^{-2 \sigma-1} d \xi=O(1 / c),
$$

as desired.

Conversely, assume

$$
K_{\sigma+1 / 2}(c)=O(1 / c)
$$

we wish to show that then $I_{\sigma+1 / 2}(c)=O(1 / c)$. But if (7.16) holds, then

$$
\int_{|\xi|>c}\left|\widehat{Q}_{0}(x,-\xi)\right|^{2}|\xi|^{-2 \sigma-1} d \xi=\int_{|\xi|>c}\left|\sum_{\lambda} \overline{\widehat{\psi}}^{\lambda}(-\xi) Z^{\lambda}(x, \xi)\right|^{2}|\xi|^{-2 \sigma-1} d \xi=O(1 / c),
$$


$(c \rightarrow 0)$, by the boundedness of $Z^{\lambda}(x, \xi)$. Then by Proposition 4.1, (7.14) holds, and so by Theorem 5.1 the best order of approximation in $H^{s}$ is $r=\sigma-d / 2$. Thus by $(i v)$ of this theorem, which has already been proved, it follows that

$$
I_{\sigma+1 / 2}(c)=O(1 / c),
$$

as desired. This completes the proof of $(v)$.

Proof of Corollary 6: The implication $(a) \Rightarrow(b)$ is clear, while $(b) \Rightarrow(c)$ and $(b) \Rightarrow(d)$ follow from Theorems 1 through 3. Also $(a)$ implies that $\sigma=0$, which by Theorem 5 implies $(e)$. Thus $(a)$ implies $(b),(c),(d)$, and $(e)$. On the other hand, by Proposition 1.8, $(b)$ implies $\sigma=0$ and thus $(a)$. By Theorems 1 through 3, $(c)$ and $(d)$ imply $I-P: H^{s} \rightarrow L^{\infty}$ is unbounded for $s>d / 2$, which implies $(b)$ and hence also $(a)$ (recall $E=I-P: H^{s} \rightarrow L^{\infty}$ is unbounded for $0 \leq s \leq d / 2$ ). In addition, by Theorem 1 , (e) implies $(b)$ and hence $(a)$.

\section{Proof of Theorem 8}

Proof of Theorem 8: Note that the statement of the theorem yields positive convergence rates only if $t>d$, which we assume throughout. Our assumption easily implies that uniformly in $x$ and $y$,

$$
|P(x, y)| \leq K|x-y|^{-t}
$$

Indeed, since $P(x+1, y+1)=P(x, y)$ it suffices to check this for $x$ in the unit cube $\mathcal{C}$. For $x \in \mathcal{C}$, since $|\phi(x-k)| \leq(1+|k|)^{-t}$, we have

$$
\begin{aligned}
|P(x, y)| & =\left|\sum_{k} \phi(x-k) \bar{\phi}(y-k)\right| \\
& \leq K_{1} \sum_{k}(1+|k|)^{-t}(1+|y-k|)^{-t} \\
& =K_{1}\left(\sum_{|y-k| \leq|y| / 2}+\sum_{|y-k|>|y| / 2}\right)(1+|k|)^{-t}(1+|y-k|)^{-t} \\
& \leq K_{2}\left(\begin{array}{c}
|y|^{-t} \sum_{|y-k| \leq|y| / 2}(1+|y-k|)^{-t}+|y|^{-t} \sum_{|y-k|>|y| / 2}(1+|k|)^{-t}
\end{array}\right) . \\
& \leq K_{3}|y|^{-t},
\end{aligned}
$$

recalling that $t>d$. Then (8.1) follows from the boundedness of $P(x, y)$.

In this case it suffices without loss to find a uniform local rate of convergence in the unit ball $B \subset \mathbb{R}^{d}$, since the same rate will hold in any other unit ball. We consider $f \in H_{u l}^{s}$ with $\|f\|_{H_{u l}^{s}}=1$. For such an $f$ we write $f=f_{1}+f_{2}$, where $f_{1}$ is supported in $3 B$, $f_{2}$ is supported outside $2 B$, and $\left\|f_{1}\right\|_{H^{s}} \leq C$ with $C$ independent of $f$. Then 


$$
\left\|E_{n} f\right\|_{\infty} \leq\left\|E_{n} f_{1}\right\|_{\infty}+\left\|E_{n} f_{2}\right\|_{\infty}
$$

The first term satisfies

$$
\left\|E_{n} f_{1}\right\|_{\infty} \leq K_{1} \cdot 2^{-r n}
$$

with $K_{1}$ independent of $f$. The second term satisfies (for $x \in B$ )

$$
\begin{aligned}
\left|E_{n} f_{2}\right| & =\left|\int 2^{n d} P\left(2^{n} x, 2^{n} y\right) f_{2}(y) d y\right| \\
& =\left|\int 2^{n d} P\left(2^{n} x-\left[2^{n} x\right], 2^{n} y-\left[2^{n} x\right]\right) f_{2}(y) d y\right| \\
& \leq K \int_{2 \widetilde{B}} 2^{n d}\left|P\left(2^{n} x-\left[2^{n} x\right], 2^{n} y-\left[2^{n} x\right]\right)\right| d y \\
& =K \int_{2^{n+1} \widetilde{B}-\left[2^{n} x\right]}\left|P\left(2^{n} x-\left[2^{n} x\right], y\right)\right| d y \\
& \leq K_{1} \int_{2^{n+1} \widetilde{B}-\left[2^{n} x\right]}|y|^{-t} d y \\
& \leq K_{1} \int_{2^{n} \widetilde{B}}|y|^{-t} d y \\
& \leq K_{2} 2^{-n(t-d)} ;
\end{aligned}
$$

above $[\cdot]$ denotes the greatest integer function applied componentwise to vectors, and $\widetilde{B}$ is the complement of $B$. We have used the fact that $f_{2} \in L^{\infty}$, with norm $K$ bounded by the norm $\left\|f_{2}\right\|_{H_{u l}^{s}}$, since $H_{u l}^{s} \subseteq L^{\infty}$, given $s>d / 2$.

\section{Proof of Corollary 9:}

(a) The assumptions of the Corollary imply $\sigma-d / 2 \leq t-d$. For $s>\sigma$, therefore, the best order of convergence in $H_{u l}^{s}$ is the same as in $H^{s}$, i.e., $r=(\sigma-d / 2)^{(-)}$, by Theorem 8 , where $(-)$ indicates the possibility of - or its absence in the superscript. Since this statement is independent of $s>\sigma$, we conclude the optimal order of convergence is $(\sigma-d / 2)^{(-)}$.

(b) For any $1 \leq p_{1,}, p_{2} \leq \infty$ and $s_{2}>0$, the inclusion $L_{s_{1}, u l}^{p_{1}} \subseteq L_{s_{2}, u l}^{p_{2}}$ holds for $s_{1}$ sufficiently large. Indeed in this case $\|f\|_{L_{s_{1}, u l}^{p_{1}}} \geq K\|f\|_{L_{s_{2}, u l}^{p_{2}}}$ is clear from the standard Sobolev inclusion relations. In particular, $L_{s_{1}, u l}^{p} \subseteq H_{u l}^{s_{2}}$ for $s_{1}$ sufficiently large, so in the scale $\left\{L_{s_{1}}^{p}\right\}_{s_{1} \geq 0}$, the optimal order of convergence is at least that in the scale $\left\{H_{u l}^{s}\right\}_{s>0}$ and thus the scale $\left\{H^{s}\right\}_{s>0}$, as seen above. The reverse inclusion (for sufficiently large $s_{2}$ ) shows that it cannot be greater, and so is the same as in the scale $\left\{H^{s}\right\}$.

Proof of Proposition 10: This proposition follows immediately from Theorem 5, since on any compact $K$ a function in $H_{l o c}^{s}$ is a restriction of a function in $H^{s}$, and convergence 
properties for a function $f$ on $K$ depend only on the properties of $f$ in $K$, since the wavelet is compactly supported.

We conclude by remarking that most of the present results and their variations hold in general spaces of functions to which global versions of these theorems apply.

Acknowledgments: The first author thanks the U.S. Fulbright Commission and the University of Warsaw for its support. The second author is grateful to Anneli and Peter Lax for encouragement and useful comments on this work, and Louis Nirenberg for an invitation to spend a year at the Courant Institute.

\section{References}

[D1] Daubechies, I., Orthonormal bases of compactly supported wavelets, Comm. Pure Appl. Math. 41 (1988), 909-996.

[D2] Daubechies, I., Ten Lectures on Wavelets, CBMS-NSF Series in Applied Mathematics, SIAM, 1992.

[GK1] Gurarie, David, and Mark A. Kon, Radial bounds for perturbations of elliptic operators, J. Functional Analysis 56 (1984), 99-123.

[GK2] Gurarie, David, and Mark A. Kon, Resolvents and regularity properties of elliptic operators, in Operator Theory: Advances and Applications, C. Apostol, Ed., Birkhauser Verlag, 1983.

[HW] Hernandez, E. and G. Weiss, First Course in Wavelets, CRC Press, 1996.

[KKR1] Kelly S., M. Kon, and L. Raphael, Local convergence of wavelet expansions, J. Functional Analysis 126 (1994), 102-138.

[KKR2] Kelly, S., M. Kon, and L. Raphael, Pointwise convergence of wavelet expansions, Bull. Amer. Math. Soc. 30 (1994), 87-94.

[KR1] Kon, M. and L. Raphael, Convergence rates of multiresolution and wavelet expansions, to appear in Wavelet Transforms and Time-Frequency Signal Analysis, CBMS Conference Proceedings, L. Debnath, Ed., Chapter 2.

[KR2] Kon, M and L. Raphael, Characterizing convergence rates for multiresolution approximations, in Signal and Image Representation in Combined Spaces, J. Zeevi and Ronald Coifman, Eds., 1998, 415-437.

[Ma] Mallat, S., Multiresolution approximation and wavelets, Trans. Am. Math. Soc. 315 (1989), 69-88. 
[Me] Meyer, Yves, Ondelettes, Hermann, Paris, 1990.

[Si] Singer, I., The Theory of Best Approximation in Functional Analysis, CBMS Conferences in Applied Mathematics, vol. 13, SIAM, 1974.

[SF] Strang, G. and Fix, G., A Fourier analysis of the finite element variational method, in Constructive Aspects of Functional Analysis, Edizioni Cremonese, Rome, 1973.

[Wa] Walter, G., Approximation of the delta function by wavelets, J. Approximation Theory 71 (1992), 392-343.

[Wo] P. Wojtaszczyk, A Mathematical Introduction to Wavelets, Cambridge University Press, Cambridge, 1997.

\author{
Mark A. Kon \\ Department of Mathematics \\ Boston University \\ Boston, MA 02215 \\ mkon@math.bu.edu \\ Louise A. Raphael \\ Department of Mathematics \\ Howard University \\ Washington, DC 20059 \\ lar@scs.howard.edu
}


Note: This appendix is not included in the published version of this paper.

\section{Appendix for "A Characterization of Wavelet Convergence in Sobolev Spaces" by Kon and Raphael}

This appendix contains the technical proofs of statements in Section 3 of this paper.

The following is a standard fact whose proof (using the closed graph theorem) we omit. Below $B^{d}$ again denotes the unit ball of $\mathbb{R}^{d}$.

Lemma 9.1: On a normed linear space (NLS) $X$, two norms in which $X$ is complete are equivalent if whenever $f_{n} \rightarrow 0$ in the first norm and $\left\{f_{n}\right\}$ converges in the second norm, then $f_{n} \rightarrow 0$ in the second norm.

Recall a seminorm $\|\cdot\|$ on a vector space $X$ satisfies $\|\alpha f\|=|\alpha|\|f\|$ for $f \in X$ and $\alpha \in \mathbb{C}$, and satisfies the triangle inequality, but not necessarily positive definiteness. We say seminorm $\|\cdot\|$ on a vector space $X$ is complete if for every Cauchy sequence $\left\{f_{n}\right\}$ there exists $f \in X$ such that $f_{n} \rightarrow f$, i.e., $\left\|f_{n}-f\right\| \rightarrow 0$.

Note that seminorm convergence $f_{n} \rightarrow f$ does not in general determine $f$ uniquely.

Lemma 9.2: A nonnegative seminorm $\|\cdot\|$ on a vector space $X$ is complete if and only if whenever a sequence $\left\{f_{n}\right\}$ is absolutely convergent (i.e., $\sum_{n}\left\|f_{n}\right\|<\infty$ ), it follows $\sum_{n} f_{n}$

converges (i.e., there exists $f \in X$ such that $\left\|\sum_{n} f_{n}-f\right\| \rightarrow 0$ ).

Lemma 9.3: Let $\left\{\|\cdot\|_{\tau}\right\}_{\tau \in T}$ be a family of seminorms on a vector space $X$. Assume that for any $\left\{f_{n}\right\} \subset X$, if for each $\tau, f_{n} \rightarrow \infty h_{\tau} \in X$ in seminorm $\tau$ and $\sup _{\tau}\left\|h_{\tau}\right\|_{\tau}<\infty$, then there is an $h \in X$ such that $f_{n}{ }_{n \rightarrow \infty} h$ in all the seminorms $\|\cdot\|_{\tau}$. Then if $X$ is complete in each of these seminorms, it is also complete in the seminorm $\|f\| \equiv \sup _{\tau}\|f\|_{\tau}$, assuming the latter is always finite.

Proof of Lemma 3.2: (a) The forward implication is clear, and we prove the reverse. First assume $\alpha>0$. Then if $|x|<1 / 2$, we have

$$
\begin{aligned}
A(x) & \leq\left|\sum_{i=0}^{N}\left[A\left(2^{i} x\right)-A\left(2^{i+1} x\right)\right]\right|+A\left(2^{N+1} x\right) \\
& \leq C_{2} \sum_{i=0}^{N}\left|2^{i+1} x\right|^{-\alpha}+A\left(2^{N+1} x\right) \\
& \leq\left(2^{-\alpha} C_{2} /\left(1-2^{-\alpha}\right)\right)|x|^{-\alpha}+A\left(2^{N+1} x\right),
\end{aligned}
$$


where $N=N(x)$ is the largest integer such that $\left|2^{N+1} x\right|$ is less than 1 . This yields

$$
A(x) \leq C_{1}|x|^{-\alpha},
$$

with $C_{1}=2^{-\alpha} C_{2} /\left(1-2^{-\alpha}\right)+\sup _{1 / 2 \leq|x| \leq 1} A(x)$, where we note that by the definition of $N$, $1 / 2 \leq\left|2^{N+1} x\right|<1$. Also note $A\left(2^{N+1} x\right)|x|^{-\alpha} \geq A\left(2^{N+1} x\right)$ since $|x| \leq 1$. On the other hand, if $1 / 2 \leq|x|<1$, then (9.2) also clearly holds, yielding the desired bound for all $x \in B^{d}$.

On the other hand if $\alpha<0$

$$
|A(x)|=\left|\sum_{i=0}^{\infty} A\left(2^{-i} x\right)-A\left(2^{-i-1} x\right)\right| \leq C_{2} \sum_{i=0}^{\infty}\left|2^{-i} x\right|^{-\alpha}=C_{2}|x|^{-\alpha} /\left(1-2^{\alpha}\right),
$$

yielding $C_{1}=C_{2} /\left(1-2^{\alpha}\right)$.

(b) In this case

$$
\begin{aligned}
|A(x)-A(0)| & =\lim _{N \rightarrow \infty}\left|\sum_{i=0}^{N}\left(A\left(2^{-i} x\right)-A\left(2^{-i-1} x\right)\right)+A\left(2^{-N-1} x\right)-A(0)\right| \\
& =\left|\sum_{i=0}^{\infty} A\left(2^{-i} x\right)-A\left(2^{-i-1} x\right)\right| \\
& \leq C_{2}|x|^{-\alpha} /\left(1-2^{\alpha}\right),
\end{aligned}
$$

where the last inequality follows as in (9.3).

(c) Here we again need only prove the reverse implication. Our assertion is equivalent to showing that if $|A(x)-A(x / 2)| \leq D_{1}(x)|x|^{-\alpha}$ where $D_{1}(x)$ is positive with $D_{1}(x) \underset{x \rightarrow 0}{\longrightarrow}$ 0 , then $A(x) \leq D_{2}(x)|x|^{-\alpha}$, where $D_{2}$ has the same properties as $D_{1}$.

First consider the case $\alpha>0$. Then under our assumptions

$$
\begin{aligned}
A(x) & \leq\left|\sum_{i=0}^{N}\left[A\left(2^{i} x\right)-A\left(2^{i+1} x\right)\right]\right|+A\left(2^{N+1} x\right) \\
& \leq C_{2} \sum_{i=0}^{N} D_{1}\left(2^{i+1} x\right)\left|2^{i+1} x\right|^{-\alpha}+A\left(2^{N+1} x\right) .
\end{aligned}
$$

where $N$ is chosen as above. We now redefine $N(x)$ to remain an integer for all $x$, but so that as $x \rightarrow 0$ we have $K_{1} x^{1 / 2} \leq 2^{N+1} x \leq K_{2} x^{1 / 2}$. Then by part (a), since we know at least that $A(x)=O\left(|x|^{-\alpha}\right)$,

$$
A\left(2^{N+1} x\right) \leq K_{3}\left|2^{N+1} x\right|^{-\alpha} \leq K_{4}|x|^{-\alpha / 2}=o\left(|x|^{-\alpha}\right) .
$$

Consider the ratio (recall $N=N(x)$ ) 


$$
\begin{aligned}
\frac{\left.\left|\sum_{i=0}^{N} D_{1}\left(2^{i+1} x\right)\right| 2^{i+1} x\right|^{-\alpha} \mid}{2^{-\alpha}|x|^{-\alpha} \frac{1-2^{-\alpha(N+1)}}{1-2^{-\alpha}}} & =\frac{\left.\left|\sum_{i=0}^{N} D_{1}\left(2^{i+1} x\right)\right| 2^{i+1} x\right|^{-\alpha} \mid}{\sum_{i=0}^{N}\left|2^{i+1} x\right|^{-\alpha}} . \\
& \leq C_{2} \sup _{0 \leq i \leq N} D_{1}\left(2^{i+1} x\right) \\
& \longrightarrow 00
\end{aligned}
$$

Thus $A(x)=o\left(|x|^{-\alpha}\right)$, as desired, if $\alpha>0$.

Now assume $\alpha<0$, and that $A(0)=0$. In that case,

$$
\begin{aligned}
|A(x)| & =\left|\sum_{i=0}^{\infty} A\left(2^{-i} x\right)-A\left(2^{-i-1} x\right)\right|, \\
& \leq C_{2} \sum_{i=0}^{\infty} D_{1}\left(2^{-i} x\right)\left|2^{-i} x\right|^{-\alpha} \\
& =\sup _{1 \leq i<\infty} D_{1}\left(2^{-i} x\right) C_{2}|x|^{-\alpha} /\left(1-2^{\alpha}\right) \\
& =o\left(|x|^{-\alpha}\right),
\end{aligned}
$$

as desired.

Below is a more general version of Theorem 3.4, with proof included.

Theorem 3.4: The following statements are equivalent for $\alpha>0$ and a positive function $f(x)$ on the unit ball $B^{d}$ of $\mathbb{R}^{d}$ with $0<c<1$ (where all integrals are restricted to the unit ball):

(a) The integral $\int_{|x|>c} d x f(x)=O\left(c^{-\alpha}\right)$.

(b) $\int_{c / 2<|x|<c} d x f(x)=O\left(c^{-\alpha}\right)$.

(c) $\int_{c / 2<|x|<c} d x|x|^{\alpha} f(x)=O(1)$.

(d) For some (or all) $\beta<\alpha, \int_{|x|>c} d x f(x)|x|^{\beta}=O\left(c^{\beta-\alpha}\right)$

$\left[\right.$ and for $\left.\beta>\alpha, \int d x f(x)|x|^{\beta}<\infty\right]$.

(d') For some (or all) $\beta \in \mathbb{R}, \int_{c / 2<|x|<c} d x f(x)|x|^{\beta}=O\left(c^{\beta-\alpha}\right)$.

$\left(d^{\prime \prime}\right)$ For some (or all) $\beta>\alpha, \int_{|x|<c} d x f(x)|x|^{\beta}=O\left(c^{\beta-\alpha}\right)$.

$\left(d^{\prime \prime \prime}\right)$ For some (or all) $\beta>\alpha$, and some (or all) $\gamma$ with $\gamma+\beta-\alpha<0$, $\int d x f(x)|x|^{\beta}(c+|x|)^{\gamma}=O\left(c^{\gamma+\beta-\alpha}\right)$

$\left[\right.$ and for $\beta>\alpha$ and $\gamma+\beta-\alpha>0$, then $\lim _{c \rightarrow 0} \int d x f(x)|x|^{\beta}(c+|x|)^{\gamma}$ exists and is finite].

$\left(d^{\prime \prime \prime \prime}\right)$ For some (or all) $\beta, \gamma \in \mathbb{R}, \int_{c / 2<|x|<c} d x f(x)|x|^{\beta}(c+|x|)^{\gamma}=O\left(c^{\gamma+\beta-\alpha}\right)$.

(e) For any function $g(|x|) \in \mathcal{L}_{C}$ such that $\int d x|x|^{-d} g(|x|)<\infty$, it follows that $\int d x g(|x|)|x|^{\alpha} f(x)<\infty$, for some (or all) $C>1$.

Statements in brackets $[\cdot]$ may be included or excluded without changing the equivalences. 
In addition, $O(\cdot)$ may be replaced by $o(\cdot)$ simultaneously in all of the above statements excluding $(c)$, and the equivalences of $(a)-\left(b^{\prime \prime}\right)$ (i.e. all statements excluding $(c)$ ) continue to hold.

Proof of Theorem 3.4: We first prove the statements above are equivalent without the bracketed inclusions. Whenever we apply Lemma 3.2, we implicitly extend $f(x)$ to have value 0 for $|x|>1$. That (a) $\Leftrightarrow$ (b) follows from Lemma 3.2, choosing $A(c)=\int_{|x|>c} d x f(x)$. To show (b) $\Rightarrow$ (c) assume (b) holds. Then

$$
\int_{c / 2 \leq|x| \leq c} d x c^{\alpha} f(x)=O(1)
$$

However,

$$
\int_{c / 2 \leq|x| \leq c} d x(c / 2)^{\alpha} f(x) \leq \int_{c / 2 \leq|x| \leq c} d x|x|^{\alpha} f(x) \leq \int_{c / 2 \leq|x| \leq c} d x c^{\alpha} f(x)
$$

so (c) follows. This argument can be reversed to yield (c) $\Rightarrow$ (b).

The equivalence of $(c)$ and $\left(d^{\prime}\right)$ is proved in essentially the same way as that of $(b)$ and (c).

To show $(c) \Rightarrow(e)$ assume $(c)$ holds and let $C>0$. Assume $\int d x|x|^{-d} g(|x|)<\infty$ for some $g(|x|) \in \mathcal{L}_{C}$. Letting $s_{k}=\sup _{2^{-k} \leq \rho \leq 2^{-k+1}} g(\rho)$, and $i_{k}$ the same quantity with sup replaced by inf,

$$
\begin{aligned}
i_{k} \int_{2^{-k} \leq|x| \leq 2^{-k+1}} d x|x|^{\alpha} f(x) & \leq \int_{2^{-k} \leq|x| \leq 2^{-k+1}} d x g(|x|)|x|^{\alpha} f(x) \\
& \leq s_{k} \int_{2^{-k} \leq|x| \leq 2^{-k+1}} d x|x|^{\alpha} f(x) .
\end{aligned}
$$

Since $g(|x|) \in \mathcal{L}_{C}$, we have for some $K>1,1 / K \leq g(b \rho) / g(\rho) \leq K$ for $1 \leq b \leq 2$.

Thus if $|\Omega|$ is the surface area of the unit sphere in $d$ dimensions,

$$
\begin{aligned}
s_{k} & \leq K i_{k} \\
& \leq K \cdot 2^{k} \int_{2^{-k}}^{2^{-k+1}} d \rho g(\rho) \\
& =K \frac{2^{k}}{|\Omega|} \int_{2^{-k} \leq|x| \leq 2^{-k+1}} d x|x|^{1-d} g(|x|) \\
& \leq \frac{2 K}{|\Omega|} \int_{2^{-k} \leq|x| \leq 2^{-k+1}} d x|x|^{-d} g(|x|) .
\end{aligned}
$$

Thus 


$$
\begin{aligned}
\int d x g(|x|)|x|^{\alpha} f(x) & =\sum_{k=1}^{\infty} \int_{2^{-k} \leq|x| \leq 2^{-k+1}} d x g(|x|)|x|^{\alpha} f(x) \\
& \leq \sum_{k=1}^{\infty} s_{k} \int_{2^{-k} \leq|x| \leq 2^{-k+1}} d x|x|^{\alpha} f(x) \\
& \leq\left(\sup _{k \geq 1} \int_{2^{-k} \leq|x| \leq 2^{-k+1}} d x|x|^{\alpha} f(x)\right) \sum_{k=1}^{\infty} \frac{2 K}{|\Omega|} \int_{2^{-k} \leq|x| \leq 2^{-k+1}} d x|x|^{-d} g(|x|) \\
& =\left(\sup _{k \geq 1} \int_{2^{-k} \leq|x| \leq 2^{-k+1}} d x|x|^{\alpha} f(x)\right) \cdot \frac{2 K}{|\Omega|} \int d x|x|^{-d} g(|x|) \\
& <\infty,
\end{aligned}
$$

implying $(e)$.

To show $(e) \Rightarrow(c)$, assume $(e)$ for some $C>1$. We wish to show $\int_{c / 2 \leq|x| \leq c} d x|x|^{\alpha} f(x)=O(1)$, or equivalently $b_{k} \equiv \int_{2^{-k} \leq|x| \leq 2^{-k+1}} d x|x|^{\alpha} f(x)=O(1)$ as the integer $k$ becomes large. For this it suffices to show that for any positive summable sequence $\left\{a_{k}\right\}$, the sequence

$$
\left\{a_{k} \int_{2^{-k} \leq|x| \leq 2^{-k+1}} d x|x|^{\alpha} f(x)\right\}
$$

is also summable. Further it suffices to show the collection of sequences for which this holds includes the summable sequences $\left\{a_{k}\right\}$ satisfying

$$
1 / C \leq a_{k+1} / a_{k} \leq C .
$$

Indeed if $b_{k} \neq O(1)$ (i.e., is unbounded), let $\left\{b_{k_{n}}\right\}$ be a subsequence satisfying $b_{k_{n}} \geq 4 b_{k_{n-1}}$. We could then choose a family of sequences $\left\{c_{l k}\right\}_{k}$, defined by $c_{l k}=2^{-l} C^{-\left|k-k_{l}\right|}$, and then define $a_{k}=\sum_{l} c_{l k}$. Since for each $l$ the sequence $c_{l k}$ (as a function of $k$ ) satisfies (9.6), it follows that $a_{k}$ does as well. Furthermore, clearly $a_{k}$ is summable. And finally we would have

$$
\sum_{k} a_{k} b_{k} \geq \sum_{n} a_{k_{n}} b_{k_{n}} \geq \sum_{n}\left(\sum_{l} c_{l k_{n}}\right) 4^{n} b_{k_{0}} \geq \sum_{n} c_{n k_{n}} 4^{n} b_{k_{0}}=\sum_{n} 2^{-n} 4^{n} b_{k_{0}}=\infty .
$$

Thus it would be false that (9.5) is summable for all $\left\{a_{n}\right\}$ satisfying (9.6). This shows that it suffices to show (9.5) is summable for all summable $\left\{a_{k}\right\}$ satisfying (9.6).

Given an arbitrary summable $\left\{a_{k}\right\}$ satisfying (9.6), there exists a function $g(|x|): \mathbb{R}^{d} \rightarrow \mathbb{R}$ such that $a_{k}=\int_{2^{-k} \leq|x| \leq 2^{-k+1}} d x|x|^{-d} g(|x|)$. Indeed let $g$ be chosen so $\rho^{-1} g(\rho)$ is constant on each dyadic interval $2^{-k} \leq \rho \leq 2^{-k-1}$ and equals $2^{k} a_{k} /|\Omega|$, where $\Omega$ is the surface of the unit $d$-ball. In this case 


$$
\int_{2^{-k} \leq|x| \leq 2^{-k+1}} d x|x|^{-d} g(|x|)=|\Omega| \int_{2^{-k} \leq \rho \leq 2^{-k+1}} d \rho \rho^{-1} \rho 2^{k} a_{k} /|\Omega|=a_{k} .
$$

Then

$$
\int d x|x|^{-d} g(|x|)=\sum_{k=1}^{\infty} a_{k}<\infty .
$$

For any constant $1 \leq b \leq 2$ we have

$$
\rho^{-1} g(\rho)=2^{k} a_{k} /|\Omega|
$$

for $2^{-k} \leq \rho \leq 2^{-k+1}$, while $(b \rho)^{-1} g(b \rho)=2^{k} a_{k} /|\Omega|$ or $2^{k-1} a_{k-1} /|\Omega|$. Then

$$
b^{-1} \frac{g(b \rho)}{g(\rho)}=\frac{(b \rho)^{-1} g(b \rho)}{\rho^{-1} g(\rho)}=\left\{\begin{array}{l}
\frac{2^{k-1} a_{k-1}}{2^{k} a_{k}} \\
\text { or } 1
\end{array},\right.
$$

while

$$
\frac{1}{2 C} \leq\left\{\begin{array}{c}
\frac{2^{k-1} a_{k-1}}{2^{k} a_{k}} \\
\text { or } 1
\end{array} \leq \max (C / 2,1)\right.
$$

implying that for $1 \leq b \leq 2$

$$
\frac{1}{2 C} \leq b^{-1} g(b \rho) / g(\rho) \leq \max (C / 2,1),
$$

and so

$$
\frac{1}{2 C} \leq g(b \rho) / g(\rho) \leq \max (C, 2)
$$

Further, letting $b=2$ above, we have $(2 \rho)^{-1} g(2 \rho)=2^{k-1} a_{k-1} /|\Omega|$, so

$$
\frac{(2 \rho)^{-1} g(2 \rho)}{\rho^{-1} g(\rho)}=\frac{2^{k-1} a_{k-1}}{2^{k} a_{k}}
$$

and so

$$
\frac{1}{2 C} \leq \frac{(2 \rho)^{-1} g(2 \rho)}{\rho^{-1} g(\rho)} \leq \frac{C}{2},
$$

and

$$
\frac{1}{C} \leq \frac{g(2 \rho)}{g(\rho)} \leq C
$$

so $g(\rho) \in \mathcal{L}_{C}$. 
Since we are assuming $(e)$,

$$
\begin{aligned}
\sum_{k=1}^{\infty} a_{k} \int_{2^{-k} \leq|x| \leq 2^{-k+1}} d x|x|^{\alpha} f(x) & =\sum_{k=1}^{\infty} \int_{2^{-k} \leq|x| \leq 2^{-k+1}} d x 2^{-k}|x|^{-1} g(|x|)|\Omega||x|^{\alpha} f(x) \\
& \leq \sum_{k=1}^{\infty} \int_{2^{-k} \leq|x| \leq 2^{-k+1}} d x|x||x|^{-1} g(|x|)|\Omega||x|^{\alpha} f(x) \\
& <\infty
\end{aligned}
$$

proving (9.5) is summable for an arbitrary summable sequence $\left\{a_{k}\right\}$ satisfying (9.6). Thus

$$
\int_{2^{-k} \leq|x| \leq 2^{-k+1}} d x|x|^{\alpha} f(x)=O(1),
$$

implying $(c)$, and showing $(e) \Rightarrow(c)$.

We have thus showed equivalence of $(a),(b),(c),\left(d^{\prime}\right)$, and $(e)$. For $\beta<\alpha$, Lemma 3.2 implies $(d)$ and $\left(d^{\prime}\right)$ are equivalent. If $\left(d^{\prime}\right)$ holds for some $\beta \in \mathbb{R}$, then by the same arguments as earlier (showing equivalence of $(b)$ and $(c)$ ) it holds for all $\beta \in \mathbb{R}$, proving the equivalence of $(d)$ and $\left(d^{\prime}\right)$ if in $\left(d^{\prime}\right) \beta \geq \alpha$ as well. To show equivalence of $\left(d^{\prime \prime}\right)$ and $\left(d^{\prime}\right)$, note the implication $\left(d^{\prime \prime}\right) \Rightarrow\left(d^{\prime}\right)$ is clear. The reverse follows once we observe that if $\left(d^{\prime}\right)$ holds, then (recall $f(x)$ may be assumed 0 outside the unit ball)

$$
\begin{aligned}
\lim _{k \rightarrow \infty} \int_{|x|>2^{-k}} d x f(x)|x|^{\beta} & =\lim _{k \rightarrow \infty} \sum_{k^{\prime}=-k}^{-1} \int_{2^{k^{\prime}<|x|<2^{k^{\prime}+1}}} d x f(x)|x|^{\beta} \\
& \leq \lim _{k \rightarrow \infty} \sum_{k^{\prime}=-k}^{-1} C \cdot 2^{(\beta-\alpha) k^{\prime}}<\infty
\end{aligned}
$$

so $\int d x f(x)|x|^{\beta}<\infty$. Therefore if $A(c) \equiv \int_{|x|<c} d x f(x)|x|^{\beta}$, we have $A(0)=0$, and so by Lemma $3.2 A(c)=O\left(c^{\beta-\alpha}\right)$.

That $\left(d^{\prime \prime \prime \prime}\right)$ is equivalent to $\left(d^{\prime}\right)$ is clear since if $c / 2 \leq|x| \leq c$, then $c,|x|$, and $c+|x|$ are all of the same order.

To show $\left(d^{\prime \prime \prime}\right)$ is equivalent to $\left(d^{\prime \prime \prime \prime}\right)$ it is first clear $\left(d^{\prime \prime \prime}\right)$ implies $\left(d^{\prime \prime \prime \prime}\right)$. Now assume $\left(d^{\prime \prime \prime \prime}\right)$. Then if $\beta>\alpha$ and $\gamma+\beta-\alpha<0$, it follows that $\gamma<0$, so that

$$
\begin{aligned}
\int d x f(x)|x|^{\beta} & (c+|x|)^{\gamma}=\int_{|x|<c} d x f(x)|x|^{\beta}(c+|x|)^{\gamma}+\int_{|x|>c} d x f(x)|x|^{\beta}(c+|x|)^{\gamma} \\
\leq & c^{\gamma} \int_{|x|<c} d x f(x)|x|^{\beta}+\int_{|x|>c} d x f(x)|x|^{\beta+\gamma} \\
& =O\left(c^{\gamma+\beta-\alpha}\right)+O\left(c^{\gamma+\beta-\alpha}\right)
\end{aligned}
$$

as desired, where we have used $(d)$ and $\left(d^{\prime \prime}\right)$. Thus the $(a)-(e)$ are equivalent.

To prove that we may also include the bracketed statements in $(d)$ and $\left(d^{\prime \prime \prime}\right)$, it suffices to prove the statement in brackets in $(d)$ follows from the unbracketed statement there, and similarly for $\left(d^{\prime \prime \prime}\right)$. First assume the initial part of $(d)$ holds, i.e., that for some (or 
all) $\beta<\alpha, \int_{|x|>c} d x f(x)|x|^{\beta}=O\left(c^{\beta-\alpha}\right)$. Then by what has already been proved (i.e., the equivalences of the unbracketed statements), if $\beta>\alpha, \int_{|x|<c} d x f(x)|x|^{\beta}=O\left(c^{\beta-\alpha}\right)$, so $\int d x f(x)|x|^{\beta}<\infty$. Similarly, if the initial part of $\left(d^{\prime \prime \prime}\right)$ holds and $\gamma+\beta-\alpha>0$, then we have by the dominated convergence theorem

$$
\int d x f(x)|x|^{\beta}(c+|x|)^{\gamma} \underset{c \rightarrow 0}{\longrightarrow} \int d x f(x)|x|^{\beta+\gamma}<\infty,
$$

where the right side is finite by the fact that the bracketed part of $(d)$ holds as shown above. Note the left side is always finite if $\beta>\alpha$, again by the bracketed part of $(d)$. This completes the proof of the equivalence of statements $(a)-(e)$.

To complete the proof we now assume statements $(\mathrm{a})-\left(\mathrm{d}^{\prime \prime \prime}\right)$ have $O(\cdot)$ replaced by $o(\cdot)$. The equivalence of (a) and (b) then follows directly from Lemma 3.2(c). The equivalence of parts (b), (c), (d'), and (d"') follows from the fact that, multiplied by appropriate powers of $c$, the left sides of the expressions in all of these parts have the same order (i.e., are equivalent as functions of $c$ ). The equivalence of (d) and (d') is proved in the same way as that of (a) and (b). The equivalence of (d') and (d") again follows from Lemma 3.2(c). To show that $\left(\mathrm{d}^{\prime \prime \prime}\right)$ and $\left(\mathrm{d}^{\prime \prime \prime}\right)$ are equivalent, it is first immediate that $\left(\mathrm{d}^{\prime \prime \prime}\right) \Rightarrow\left(\mathrm{d}^{\prime \prime \prime}\right)$. To prove the reverse implication, assume that (d"') holds. Then the proof of (d"') follows identically to the proof of $\left(\mathrm{d}^{\prime \prime \prime}\right) \Rightarrow\left(\mathrm{d}^{\prime \prime \prime}\right)$ in the previous case above. This completes the proof.

Proof of Lemma 9.2: Assume $X$ is complete. If $\sum_{n}\left\|f_{n}\right\|<\infty$, let $g_{N}=\sum_{n=1}^{N} f_{n}$. Then $\left\{g_{N}\right\}$ forms a Cauchy sequence, and so since $X$ is complete there is a $g$ such that $\left\|\sum_{n=1}^{N} f_{n}-g\right\| n \rightarrow \infty$

Conversely assume that whenever $\sum_{n}\left\|f_{n}\right\|<\infty$ it follows $\sum_{n} f_{n}$ converges. Then if $\left\{f_{n}\right\}$ is a Cauchy sequence let $f_{n_{k}}$ be a subsequence satisfying $\left\|f_{n_{k}}-f_{n}\right\| \leq 2^{-k}$ for all $n>n_{k}$. Then $f_{n_{k}}=f_{n_{1}}+\sum_{2 \leq k^{\prime} \leq k}\left(f_{n_{k^{\prime}}}-f_{n_{k^{\prime}-1}}\right)$. By our assumption since the infinite sum of the norms in the previous expression is finite, $\left\|f_{n_{k}}-f\right\| \underset{k \rightarrow \infty}{\longrightarrow} 0$ for some $f \in X$. Thus by the triangle inequality $\left\|f_{n}-f\right\|_{n \rightarrow \infty} 0$ and $X$ is complete.

Proof of Lemma 9.3: Assume these hypotheses and let $\sum_{n}\left\|f_{n}\right\|<\infty$. Then for each $\tau$ $\sum_{n}\left\|f_{n}\right\|_{\tau}<\infty$, and since $\|\cdot\|_{\tau}$ is complete, $\sum_{n} f_{n}$ converges in $\|\cdot\|_{\tau}$. By our assumption $\left\|\sum_{n=1}^{N} f_{n}-f\right\|_{\tau} \underset{N \rightarrow \infty}{\rightarrow} 0$ for all $\tau$ for some fixed $f$.

Note if $g_{n} \rightarrow g$ in the $\tau$ norm, then $\left\|g_{n}\right\|_{\tau} \rightarrow\|g\|_{\tau}$. Indeed $\left\|g_{n}\right\| \leq\|g\|+\left\|g_{n}-g\right\|$, and $\|g\| \leq\left\|g_{n}\right\|+\left\|g-g_{n}\right\|$. Thus if $f=\sum_{n} f_{n}$ in $\|\cdot\|_{\tau}$ (recall such sums are not unique), then $\|f\|_{\tau} \leq \sum_{n}\left\|f_{n}\right\|_{\tau}$, since $\left\|\sum_{n=1}^{N} f_{n}\right\|_{\tau} \underset{N \rightarrow \infty}{\longrightarrow}\|f\|_{\tau}$. 
Now

$$
\begin{aligned}
\left\|\sum_{n=1}^{N} f_{n}-f\right\| & =\sup _{\tau}\left\|\sum_{n=1}^{N} f_{n}-f\right\|_{\tau} \\
& =\sup _{\tau}\left\|\sum_{n=N+1}^{\infty} f_{n}\right\|_{\tau} \\
& \leq \sup _{\tau} \sum_{n=N+1}^{\infty}\left\|f_{n}\right\|_{\tau} \\
& \leq \sum_{n=N+1}^{\infty} \sup _{\tau}\left\|f_{n}\right\|_{\tau} \\
& =\sum_{n=N+1}^{\infty}\left\|f_{n}\right\|_{N \rightarrow \infty} 0
\end{aligned}
$$

proving completeness of $\|\cdot\|$.

Proof of Corollary 3.5: Define the norms associated with the above statements as follows (subscript refers to statement):

$$
\begin{aligned}
& \|f\|_{a} \equiv \sup _{c \in[0,1]} c^{\alpha} \int_{|x|>c} d x|f(x)| ; \quad\|f\|_{b} \equiv \sup _{c \in[0,1]} c^{\alpha} \int_{c / 2<|x|<c} d x|f(x)| \\
& \|f\|_{c} \equiv \sup _{c \in[0,1]} \int_{c / 2<|x|<c} d x|x|^{\alpha}|f(x)| ; \quad\|f\|_{d} \equiv \sup _{c \in[0,1]} c^{\alpha-\beta} \int_{|x|>c} d x|f(x)||x|^{\beta} \\
& \|f\|_{d^{\prime}} \equiv \sup _{c \in[0,1]} c^{\alpha-\beta} \int_{c / 2<|x|<c} d x|f(x)||x|^{\beta} ; \quad\|f\|_{d^{\prime \prime}} \equiv \sup _{c \in[0,1]} c^{\alpha-\beta} \int_{|x|<c} d x|f(x)||x|^{\beta} \\
& \|f\|_{d^{\prime \prime \prime}} \equiv \sup _{c \in[0,1]} c^{\alpha-\beta-\gamma} \int d x|f(x)||x|^{\beta}(c+|x|)^{\gamma} ; \\
& \|f\|_{d^{\prime \prime \prime \prime}} \equiv \sup _{c \in[0,1]} c^{\alpha-\beta-\gamma} \int_{c / 2<|x|<c} d x|f(x)||x|^{\beta}(c+|x|)^{\gamma} ; \\
& \|f\|_{e} \equiv \sup _{g \in \mathcal{L}_{C}} \frac{1}{\|g\|_{\mathcal{L}}} \int d x g(|x|)|x|^{\alpha}|f(x)| .
\end{aligned}
$$

These are norms since the triangle inequality can be verified for all of them, and they are all positive definite. Further, defining as each norm's domain the space of functions $f: B^{d} \rightarrow \mathbb{C}$ on which it is finite, each of these norms has the same domain $A$ by Theorem 3.4. We claim each of these norms is complete on $A$.

The proof of completeness is similar for all the norms. To show $\|\cdot\|_{b}$ is complete for example, note $\|f\|_{b}$ is equivalent to the norm

$$
\|f\|_{b^{*}} \equiv \sup _{k \in \mathbb{Z}^{+}} 2^{-k \alpha} \int_{2^{-k-1}<|x|<2^{-k}} d x|f(x)|,
$$

where $\mathbb{Z}^{+}$denotes the nonnegative integers. Defining the seminorm $\|f\|_{b^{*}, k} \equiv 2^{-k \alpha} \int_{2^{-k-1}<|x|<2^{-k}} d x|f(x)|$, we see this seminorm is complete, being equivalent to an $L^{1}$ seminorm. In addition, if $f_{n}$ is a sequence which converges in each seminorm $b^{*}, k$ to $h_{k}$, and $\sup _{k}\left\|h_{k}\right\|_{b^{*}, k}<\infty$, then clearly there is a $h \in A$ such that $f_{n} \rightarrow h$ in the norm 
$\|\cdot\|_{b^{*}, k}, h$ being equal to $h_{k}$ in the interval $2^{-k+1}<|x|<2^{-k}$. Thus by Lemma 9.3 the norm $\|\cdot\|_{b^{*}}$ is complete, and hence so is $\|\cdot\|_{b}$.

To show $\|\cdot\|_{e}$ is complete on the domain $A$, define for each positive $g$ with $\|g\|_{\mathcal{L}}=1$ the norm $\|f\|_{e, g}=\int d x g(|x|)|x|^{\alpha}|f(x)|$. Then $A$ is complete in this norm, since it is a weighted $L^{1}$ norm. If we have $f_{n} \rightarrow h_{g}$ in each of the norms $\|\cdot\|_{e, g}$, then since each $g$ is positive, it follows that the functions $h_{g}$ must all be the same, i.e., $h_{g} \equiv h$ for some fixed $h$. Thus by Lemma $9.3\|\cdot\|_{e}$ is complete in $A$. The proofs of completeness for the other norms follow similarly.

By Lemma 9.1 in order to prove equivalence of the norms on their common domain $A$ it now suffices to show that if $f_{n} \rightarrow 0$ in one of these norms and converges in a second, then $f_{n} \rightarrow 0$ in the second norm. Since convergence in all the norms implies $L^{1}$ convergence on compacts not containing 0 , it is easy to see that the same limit must be obtained in all the norms if it exists. Thus the norms are all equivalent, proving the equivalence of (a) - (e).

To prove equivalence of (e) and (e'), note that for fixed $C$, defining the space

$$
\mathcal{K}_{C}=\left\{g \in \mathcal{L}_{C}:\|g\|_{\mathcal{L}}<\infty\right\}
$$

each $f_{q}$ defines a linear functional $F_{q}$ on $\mathcal{K}_{C}$, defined by

$$
F_{q}(g)=\int d x g(|x|)|x|^{\alpha} f_{q}(x) .
$$

By the uniform boundedness principle, the family $\left\{F_{q}\right\}$ is uniformly bounded on $\mathcal{K}_{C}$ if and only if it is uniformly bounded for each $g \in \mathcal{K}_{C}$. However, uniform boundedness of $\left\{F_{q}\right\}$ on $K_{C}$ is equivalent to (e), while uniform boundedness for each $g \in \mathcal{K}_{C}$ is equivalent to $\left(e^{\prime}\right)$, proving equivalence of $(\mathrm{e})$ and $\left(\mathrm{e}^{\prime}\right)$.

That the bracketed statement in (d) follows from the unbracketed statement follows from the fact that we have already showed that (d) implies (d"), which completes the proof.

In (d"'), if $\gamma+\beta-\alpha>0$, then it follows from (d"') that

$$
\int_{c / 2<|x|<c} d x f_{q}(x)|x|^{\beta}(c+|x|)^{\gamma} \leq K_{8} c^{\gamma+\beta-\alpha} .
$$

Summing the left side for $c=1 / 2^{n}$, with $n=0,1,2, \ldots$, we get $\int_{0}^{1} d x f_{q}(x)|x|^{\beta}(c+|x|)^{\gamma}$. On the other hand, the right side of (9.6) adds up to a finite number, giving the bracketed part of (d"'). This completes the proof. 